\title{
Orbits of Groups Generated by Transvections over $\mathbb{F}_{2}$
}

\author{
AHMET I. SEVEN* \\ Northeastern University, Boston, MA, USA
}

aseven@mystic.math.neu.edu

Received April 17, 2003; Revised September 10, 2004; Accepted September 10, 2004

\begin{abstract}
Let $V$ be a finite dimensional vector space over the two element field. We compute orbits for the linear action of groups generated by transvections with respect to a certain class of bilinear forms on $V$. In particular, we compute orbits that are in bijection with connected components of real double Bruhat cells in semisimple groups, extending results of M. Gekhtman, B. Shapiro, M. Shapiro, A. Vainshtein and A. Zelevinsky.
\end{abstract}

Keywords: transvections, real double Bruhat cells

\section{Introduction}

Let $V$ be a finite dimensional vector space over the two element field $\mathbb{F}_{2}$ with an $\mathbb{F}_{2^{-}}$ valued bilinear form $\Omega(u, v)$. For any non-zero vector $a \in V$ such that $\Omega(a, a)=0$, the transvection $\tau_{a}$ is the linear transformation defined as $\tau_{a}(x)=x+\Omega(x, a) a$ for all $x \in V$. If the form $\Omega$ is alternating, i.e. $\Omega(a, a)=0$ for all $a \in V$, then $\tau_{a}$ preserves $\Omega$, i.e. $\Omega(x, y)=\Omega\left(\tau_{a}(x), \tau_{a}(y)\right)$; in this case $\tau_{a}$ is called a symplectic transvection. Since we work over $\mathbb{F}_{2}$, each transvection $\tau_{a}$ is an involution, i.e. $\tau_{a}^{2}(x)=x$. For a linearly independent subset $B$ of $V$, we denote by $\Gamma_{B}$ the group generated by transvections $\tau_{b}$ for $b \in B$. We define $\operatorname{Gr}(B)$ as the graph whose vertex set is $B$ and $b_{i}, b_{j}$ in $B$ are connected if $\Omega\left(b_{i}, b_{j}\right)=1$ or $\Omega\left(b_{j}, b_{i}\right)=1$. In this paper, we study the linear action of $\Gamma_{B}$ in $V$ for a linearly independent subset $B$ such that $\operatorname{Gr}(B)$ is connected. For $\Omega$ which is alternating, a description of $\Gamma_{B}$-orbits were obtained in $[2,15,24]$ for $B$ such that $\operatorname{Gr}(B)$ contains the Dynkin graph $E_{6}$ as a subgraph (see figure 1). We give a description of the $\Gamma_{B}$-orbits for the remaining linearly independent subsets (Theorems 2.9, 2.13, 2.15). Furthermore, we compute $\Gamma_{B}$-orbits corresponding to a certain class of non-skew-symmetric bilinear forms (Theorem 2.16) extending the results of [8].

There is a rich literature on groups generated by symplectic transvections over $\mathbb{F}_{2}$. McLaughlin obtained a classification of irreducible linear groups generated by symplectic transvections $[18,19]$. In our set-up given above, McLaughlin's classification reads as follows: for a non-degenerate $\Omega$, the group $\Gamma_{B}$ is one of the following: a symplectic group, a symmetric group or an orthogonal group (see also [14]). In [26], McLaughlin's results are related to polar geometry via a classification of a particular type of graphs 
(see also $[21,25]$ ). McLaughlin's results also give a classification of simple $J$-systems, which may be considered as analogues of finite root systems for the theory of Lie algebras in characteristic $2[12,20]$. On the other hand, Hall obtained a classification of a class of 3-transposition groups which include certain groups generated by symplectic transvections [10,11]. Furthermore, Hall extended Shult's classification of certain partial linear spaces in polar geometry [3,10,11]. Hall also gave some group theoretical properties of symplectic transvections and discussed applications to the topology of surfaces and mapping class groups [9]. Janssen extended McLaughlin's classification to degenerate cases in [15]. Independently, Brown and Humphries obtained some of the results given by Janssen $[2,15]$. Humphries also obtained a combinatorial characterization of symplectic transvection groups which are isomorphic to symmetric groups [14].

Although symplectic transvection groups are classified, orbits of their linear actions have not been completely understood. In this paper, we give an explicit description of such orbits in the set-up given above. A description of the $\Gamma_{B}$-orbits for a basis $B$ such that $\operatorname{Gr}(B)$ is a tree was given in [2]. We extend this description to an arbitrary group of the form $\Gamma_{B}$ for a given linearly independent subset $B$ which is not necessarily a basis (Theorems 2.9, 2.13, $2.15)$. We also obtain a combinatorial characterization of a class of graphs $G r(B)$ such that the group $\Gamma_{B}$ is, in general, not isomorphic to an orthogonal group (Theorem 2.7 and [2, Theorem 8.5]).

Our interest in groups $\Gamma_{B}$ and their orbits comes from the study of double Bruhat cells initiated in $[7,17]$. A double Bruhat cell in a simply connected connected complex semisimple group $G$ is the variety $G^{u, v}=B u B \cap B_{-} v B_{-}$, where $B$ and $B_{-}$are two opposite Borel subgroups, and $u, v$ any two elements of the Weyl group $W$. They provide a geometric framework for the study of total positivity in semisimple groups. They are also closely related to symplectic leaves in the corresponding Poisson-Lie groups, see e.g. $[13,16]$ and references therein. A reduced double Bruhat cell $L^{u, v}$ is the quotient $G^{u, v} / H$ under the right or left action of the maximal torus $H=B \cap B_{-}$. It was shown in [24] and [28] that the connected components of the real part $L^{u, v}(\mathbb{R})$ are in a natural bijection with the

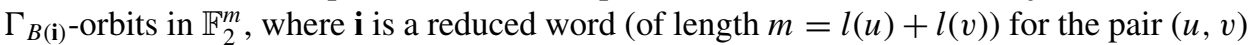
in the Coxeter group $W \times W$, and $B(\mathbf{i})$ is the corresponding set of $\mathbf{i}$-bounded indices as defined in [24]. The $\Gamma_{B(\mathbf{i})}$-orbits for simply laced (resp. non-simply-laced) groups have been computed in [24] (resp. in [8]) under the assumption that $\operatorname{Gr}(B(\mathbf{i}))$ contains the graph $E_{6}$ (figure 1). Our results are general enough to compute $\Gamma_{B(\mathbf{i}) \text {-orbits that are related to real }}$ double Bruhat cells in semisimple groups.

The groups $\Gamma_{B}$ also appeared earlier in singularity theory. To be more precise, let us assume that $B$ is a basis and $\Omega$ is an alternating form on $V$. Our connectedness assumption on $G r(B)$ implies, in particular, that $B$ is contained in a $\Gamma_{B}$-orbit, which is denoted by $\Delta$. In the language of singularity theory, the orbit $\Delta$ is called a skew-symmetric vanishing lattice

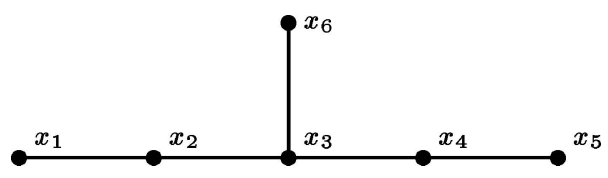

Figure 1. The Dynkin graph $E_{6}$. 
with monodromy group $\Gamma_{B}$, c.f. [15]. The main example of a skew-symmetric vanishing lattice is the Milnor lattice of an odd dimensional isolated complete intersection singularity, see e.g. [6]. A classification of monodromy groups of such lattices is given in [15]. According to this classification, if the graph $\operatorname{Gr}(B)$ contains $E_{6}$ as a subgraph, then the group $\Gamma_{B}$ has precisely two non-trivial $\Gamma_{B}$-orbits which are the sets $Q_{B}^{-1}(1)$ and $Q_{B}^{-1}(0)$, here $Q_{B}$ is the associated quadratic form [15]. To extend this results to an arbitrary basis $B$ (not necessarily containing $E_{6}$ ), we introduce a function $d: V-\{0\} \rightarrow \mathbb{Z}_{>0}$ given by

$$
d(x)=\min \left\{s: x=x_{1}+\cdots+x_{s}, \text { for some } x_{i} \in \Delta\right\} .
$$

We prove that, for arbitrary $B$, non-trivial $\Gamma_{B}$-orbits are precisely the level sets of $d$ (Theorem 2.6). We also give an explicit description of the function $d$ in terms of the graph $G r(B)$ for any basis $B$ (Theorems 2.6, 2.13). Furthermore we extend this realization to a linearly independent set $B$ which is not a basis and give an explicit description of the orbits (Theorem 2.15) extending the results of [24].

To study the action of $\Gamma_{B}$, we use combinatorial and algebraic methods. Our main combinatorial tool is a class of graph transformations generated by basic moves. More precisely, for every two elements $a, c \in B$ such that $\Omega(a, c)=1$, the basic move $\phi_{c, a}$ replaces $c$ with $\tau_{a}(c)$ and leaves other elements of $B$ unchanged. The essential feature of those moves is to preserve the associated group $\Gamma_{B}$, i.e. if $B^{\prime}$ is obtained from $B$ by a sequence of basic moves, then $\Gamma_{B}=\Gamma_{B^{\prime}}$. Basic moves were suggested to me by A. Zelevinsky; however it was brought to my attention that they had been introduced in [1]. (We thank the anonymous referee for pointing this out). It is important, e.g. in the theory of double Bruhat cells, to be able to recognize whether a given graph can be obtained from another using basic moves. Our Theorems 2.9, 2.10, 2.11, 2.13 solve this recognition problem for the classes of graphs that do not contain the subgraph $E_{6}$.

\section{Main results}

In this section, we recall some statements from $[1,2,15]$ and state our main results. As in Section 1, $V$ denotes a finite dimensional vector space over the 2-element field $\mathbb{F}_{2}$. Unless otherwise stated, $\Omega$ denotes an $\mathbb{F}_{2}$-valued alternating bilinear form on $V$. For a subspace $U$ of $V$, we denote by $U_{0}$ the kernel of the form $\Omega$ in $U$, i.e. $U_{0}=\{x \in U: \Omega(x, u)=$ 0 , for any $u \in U\}$. We assume that $B$ is a linearly independent subset whose graph $\operatorname{Gr}(B)$ is connected. By some abuse of notation, we will sometimes denote $\operatorname{Gr}(B)$ by $B$. By a subgraph of $\operatorname{Gr}(B)$, we always mean a graph $X$ obtained from $\operatorname{Gr}(B)$ by taking an induced subgraph on a subset of vertices. If $B$ is a basis, then there is a one-to-one correspondence $x \rightarrow \operatorname{Gr}(B, x)$ between $V$ and subgraphs of $\operatorname{Gr}(B)$ defined as follows: $\operatorname{Gr}(B, x)$ is the subgraph of $G r(B)$ on vertices $b_{i_{1}}, \ldots, b_{i_{k}}$, where $x=b_{i_{1}}+\cdots+b_{i_{k}}$ is the expansion of $x$ in the basis $B$. By some abuse of notation, we sometimes denote $\operatorname{Gr}(B, x)$ by $x$. We say that a vector $u$ is contained in $\operatorname{Gr}(B, x)$ if $\operatorname{Gr}(B, u)$ is contained in $\operatorname{Gr}(B, x)$.

Definition 2.1 [1, Definition 3] Let $a, c$ be in $B$ such that $\Omega(a, c)=1$. The basic move $\phi_{c, a}$ is the transformation that replaces $c \in B$ by $\tau_{a}(c)=c+a$ and keeps other elements 


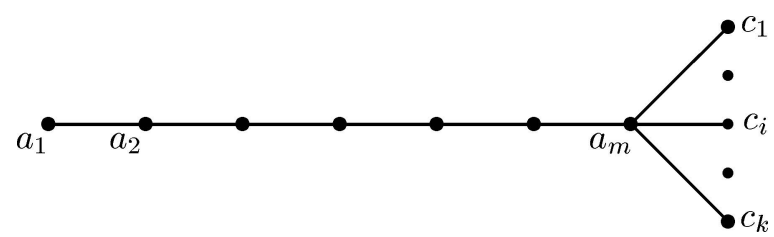

Figure 2. The graph $D_{m, k}$.

the same, i.e. $\phi_{c, a}(c)=c+a$ and $\phi_{c, a}(b)=b$ for $b \neq c$. We call two linearly independent subsets $B$ and $B^{\prime}$ equivalent if $B^{\prime}$ is obtained from $B$ by a sequence of basic moves. If $B$ and $B^{\prime}$ are equivalent, then their graphs $\operatorname{Gr}(B)$ and $\operatorname{Gr}\left(B^{\prime}\right)$ are also said to be equivalent.

We note that this equivalence relation is well-defined because $\phi_{\tau_{a}(c), a} \phi_{c, a}(B)=B$. We also note that the basic move $\phi_{c, a}$ changes $\operatorname{Gr}(B)$ as follows: suppose that $p$ and $q$ are the vertices that represent the basis vectors $a$ and $c$ respectively. The move $\phi_{c, a}$ connects $q$ to vertices that are connected to $p$ but not connected to $q$. At the same time, it disconnects vertices from $q$ if they are connected to $p$. It follows that, for any basis $B^{\prime}$ which is equivalent to $B$, the graph $\operatorname{Gr}\left(B^{\prime}\right)$ is connected. Furthermore, $\Gamma_{B}=\Gamma_{B^{\prime}}$ [1, Proposition 3.1]. Also, there exists a basis $B^{\prime}$ equivalent to $B$ such that $G r\left(B^{\prime}\right)$ is a tree [2, Theorem 3.3].

Definition 2.2 Let $m, k$ be integers such that $m \geq 2, k \geq 1$. A graph of the form in figure 2 is said to be of type $D_{m, k}$.

We note that the graph $D_{m, 1}$ is the Dynkin graph of type $A_{m+1}$ while $D_{m, 2}$ is the Dynkin graph of type $D_{m+2}$.

\subsection{Orbits of groups generated by symplectic transvections of a basis}

To describe such orbits we need to recall some basic facts from the theory of quadratic forms over $\mathbb{F}_{2}$. A quadratic form $Q$ is an $\mathbb{F}_{2}$-valued function on $V$ having the following property:

$$
Q(u+v)=Q(u)+Q(v)+g(u, v), \quad(\text { for all } u, v \in V)
$$

where $g: V \times V \rightarrow \mathbb{F}_{2}$ is an alternating bilinear form. It is clear that the quadratic form $Q$ completely determines the associated bilinear form $g$. Recall (see e.g. [5]) that there exists a symplectic basis $\left\{e_{1}, f_{1}, \ldots, e_{r}, f_{r}, h_{1}, \ldots, h_{p}\right\}$ in $V$ such that $g\left(e_{i}, f_{j}\right)=\delta_{i, j}$, and the rest of the values of $g$ are 0 ; here $\delta_{i, j}$ is the Kronecker symbol. Let us write $V_{0}=\{x \in V: g(x, v)=0$, for any $v \in V\}$. If $Q\left(V_{0}\right)=\{0\}$, then the Arf invariant of $Q$ is defined as

$$
\operatorname{Arf}(Q)=\sum Q\left(e_{i}\right) Q\left(f_{i}\right)
$$

It is well known from the theory of quadratic forms that $\operatorname{Arf}(Q)$ is independent of the choice of the symplectic basis [5]. 
Two quadratic forms $Q$ and $Q^{\prime}$ on $V$ are isomorphic if there is a linear isomorphism $T: V \rightarrow V$ such that $Q(T(x))=Q^{\prime}(x)$ for any $x \in V$. According to [4,5], isomorphism classes of quadratic forms $\{Q\}$ on $V$ are determined by their Arf invariants and their restrictions $\left\{\left.Q\right|_{V_{0}}\right\}$. More precisely, for fixed dimensions of $V$ and $V_{0}$, there exist at most 3 isomorphism classes of quadratic forms $\{Q\}$ and each isomorphism class is determined by precisely one of the following:

(i) $Q\left(V_{0}\right)=0, \operatorname{Arf}(Q)=1$

(ii) $Q\left(V_{0}\right)=0, \operatorname{Arf}(Q)=0$

(iii) $Q\left(V_{0}\right)=\mathbb{F}_{2}$

Let us now assume that $B$ is a basis of the $\mathbb{F}_{2}$-space $V$ equipped with an alternating form $\Omega$. We denote by $Q_{B}$ the unique quadratic form associated with $\Omega$ and $B$ as follows: $Q_{B}(u+v)=Q_{B}(u)+Q_{B}(v)+\Omega(u, v),(u, v \in V)$, and $Q_{B}(b)=1$ for all $b \in B$. It is easy to see that $Q_{B}$ is $\Gamma_{B}$-invariant, i.e. $Q_{B}\left(\tau_{a}(x)\right)=Q_{B}(x)$ for all $a \in B$. This also implies that quadratic forms are invariant under basic moves; i.e. if $B$ and $B^{\prime}$ are equivalent bases, then $Q_{B}(x)=Q_{B^{\prime}}(x)$ for any $x \in V$. Furthermore, the function $Q_{B}$ completely determines the $\Gamma_{B}$-orbits in $V-V_{0}$ when the graph $\operatorname{Gr}(B)$ contains a subgraph equivalent to $E_{6}$ :

Theorem 2.3 [2, Theorem 4.1; 15, Theorem 3.8] Let $V$ and $V_{0}$ have dimensions $2 n+p$ and $p$ respectively. Suppose that $B$ is a basis whose graph $\operatorname{Gr}(B)$ is equivalent to a tree which contains $E_{6}$ as a subgraph. Then $\operatorname{Gr}(B)$ is equivalent to one of the trees in figure 3, figure 4 or figure 5, depending on $Q_{B}\left(V_{0}\right)$ and $\operatorname{Arf}\left(Q_{B}\right)$. Furthermore, the group $\Gamma_{B}$ has precisely two orbits in $V-V_{0}$. They are intersections of $V-V_{0}$ with the sets $Q_{B}^{-1}(0)$ and $Q_{B}^{-1}(1)$.

Proposition 2.4 [2, Theorem 4.2] Suppose that $\operatorname{dim}(V) \geq 3$ and let $B$ be a tree that does not contain $E_{6}$. Then it is equivalent to a tree of type $D_{m, k}$, i.e. a tree of the form in figure 2 , for some $m \geq 2, k \geq 1$. Furthermore, $B$ is not equivalent to any tree $B^{\prime}$ that contains $E_{6}$.

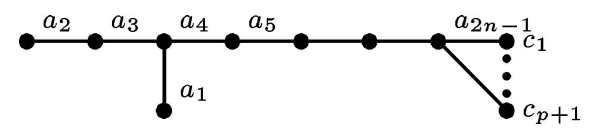

Figure 3. $\operatorname{Arf}\left(Q_{B}\right)=1$ if $n \equiv 2,3 \bmod (4), \operatorname{Arf}\left(Q_{B}\right)=0$ if $n \equiv 0,1 \bmod (4), V_{0}=$ linear span of $\left\{c_{1}+\right.$ $\left.c_{p+1}, c_{2}+c_{p+1}, \ldots, c_{p}+c_{p+1}\right\}, Q_{B}\left(V_{0}\right)=0$.

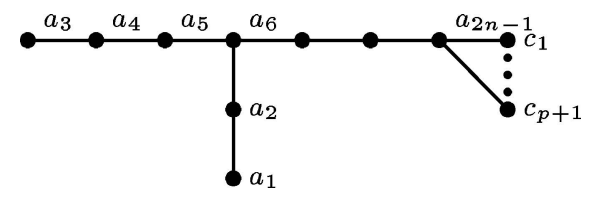

Figure 4. $\operatorname{Arf}\left(Q_{B}\right)=1$ if $n \equiv 0,1 \bmod (4), \operatorname{Arf}\left(Q_{B}\right)=0$ if $n \equiv 2,3 \bmod (4), V_{0}=$ linear span of $\left\{c_{1}+c_{p+1}, c_{2}+c_{p+1}, \ldots, c_{p}+c_{p+1}\right\}, Q_{B}\left(V_{0}\right)=0$. 


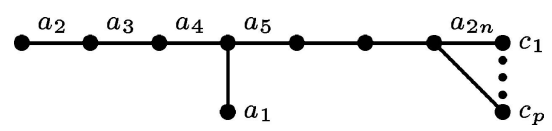

Figure 5. $Q_{B}\left(V_{0}\right)=\mathbb{F}_{2}, V_{0}=$ linear span of $\left\{c_{1}+c_{p}, c_{2}+c_{p}, \ldots, c_{p-1}+c_{p}, a_{1}+a_{2}+a_{4}\right\}$.

Thus the trees given in Figures 2-5 form a complete set of representatives for equivalence classes of graphs. For a basis $B$ whose graph is a tree of type $D_{m, k}$, there is the following description of $\Gamma_{B}$-orbits.

Theorem 2.5 [2, Theorem 7.2] Let $V$ be an $\mathbb{F}_{2}$-space with $\operatorname{dim}(V) \geq 3$ and let $B$ be $a$ basis whose graph is a tree of type $D_{m, k}$, i.e. a tree of the form in figure 2 , with $m \geq 2, k \geq 1$. Then, two vectors $x, y$ in $V-V_{0}$ lie in the same $\Gamma_{B}$-orbit if and only if $G r(B, p(x))$ and $\operatorname{Gr}(B, p(y))$ have the same number of connected components, where $p$ is the linear map on $V$ defined as $p\left(a_{i}\right)=a_{i}$ for all $i=1, \ldots, m, p\left(c_{j}\right)=c_{1}$ for $j=1, \ldots, k$.

Our next result gives a unified description of $\Gamma_{B}$ orbits.

Theorem 2.6 Let $B$ be an arbitrary basis in $V$ such that $G r(B)$ is connected and let $d: V \rightarrow \mathbb{Z}_{>0}$ be the function defined as

$$
d(x)=\min \left\{s: x=x_{1}+\cdots+x_{s}, \text { for some } x_{i} \in \Delta\right\}
$$

where $\Delta$ is the $\Gamma_{B}$-orbit that contains $B$. Let $x, y$ be vectors in $V-V_{0}$. Then $x$ and $y$ lie in the same $\Gamma_{B}$-orbit if and only if $d(x)=d(y)$. Furthermore, if $B$ is equivalent to a tree which contains $E_{6}$, then $d(x)=2-Q_{B}(x)$ for any $x \in V-V_{0}$.

In Theorem 2.13, we will obtain an explicit expression of the function $d$ for an arbitrary basis $B$ which is equivalent to a tree that does not contain $E_{6}$. Our next result allows one to recognize such bases easily.

Theorem 2.7 A connected graph $B$ is equivalent to a tree of type $D_{m, k}$ with $m \geq 2, k \geq 1$ if and only if it does not contain any subgraph which is equivalent to $E_{6}$. Furthermore, the graph $B$ is equivalent to a tree of type $D_{m, 1}$ if and only if it does not have subgraphs of the following form:

a tree of type $D_{2,2}$,

two triangles sharing a common edge,

any cycle whose length is greater than or equal to 4.

Corollary 2.12 also provides an alternate method to recognize a graph which is equivalent to a tree that does not contain $E_{6}$.

Our next theorem introduces an important class of vectors which are fixed by $\Gamma_{B}$.

Theorem 2.8 Let $B$ be an arbitrary basis in $V$ such that $G r(B)$ is connected and let $\Delta$ denote the $\Gamma_{B}$-orbit that contains $B$. Let $V_{000}$ be the set that consists of vectors $y$ in $V_{0}$ 
such that $y=x_{1}+x_{2}$ for some $x_{1}, x_{2} \in \Delta$. Then $V_{000}$ is a vector subspace of $V$ and every $\Gamma_{B}$-orbit in $V-V_{0}$ is a union of cosets in $V / V_{000}$. Furthermore, we have $\operatorname{dim}\left(V_{0} / V_{000}\right) \leq 1$.

Our next results allow one to locate all of the vectors in $V_{000}$ for a basis whose graph does not contain $E_{6}$.

Theorem 2.9 Let $B$ be a basis whose graph is equivalent to a tree of type $D_{m, k}$ with $m \geq 2, k \geq 1$. Suppose that $X$ is a subgraph which is one of the types in (2.1). Then $\mathbb{F}_{2}^{X} \cap V_{000} \neq\{0\}$, where $\mathbb{F}_{2}^{X}$ is the linear span of the vectors contained in $X$. Furthermore, if $X=\left[x_{1}, x_{2}, \ldots, x_{r}\right]$ is a cycle whose length $r$ is greater than or equal to 5 , then $\mathbb{F}_{2}^{X} \cap V_{000}$ is spanned by the vector $x_{1}+x_{2}+\cdots+x_{r}$.

Theorem 2.10 The conditions in Theorem 2.9 characterize a graph which is equivalent to a tree of type $D_{m, k}$ for some $m \geq 3, k \geq 1$. More precisely, suppose that $B$ is a basis whose graph $G r(B)$ has the following property: for any subgraph $X$ which is of one of types in (2.1), we have $\mathbb{F}_{2}^{X} \cap V_{000} \neq\{0\}$. Then $\operatorname{Gr}(B)$ is equivalent to a tree of type $D_{m, k}, m \geq 3, k \geq 1$.

Theorem 2.11 Let $B$ be a basis whose graph is equivalent to a tree of type $D_{m, k}$ with $m \geq 2, k \geq 1$. If $\operatorname{dim}(V) \geq 3$, then $V_{000}=\sum_{X} \mathbb{F}_{2}^{X} \cap V_{000}$ where $X$ runs through the subgraphs in (2.1).

Corollary 2.12 Let $B$ be a basis whose graph is equivalent to a tree of type $D_{m, k}$ with $m \geq 2, k \geq 2$. Suppose that $b$ is an arbitrary vertex contained in $\operatorname{Gr}(B, u)$ for some $u \in V_{000}$. Then the graph $\operatorname{Gr}(B-\{b\})$ is connected and it is equivalent to a tree of type $D_{m, k-1}$.

Our next result gives an explicit expression for the function $d$ defined in Theorem 2.6.

Theorem 2.13 Let $B$ be a basis which is equivalent to a tree of type $D_{m, k}$ with $m \geq 2, k \geq$ 1. Suppose that $x \in V-V_{0}$ and let $\bar{x}$ be any minimal representative in the coset $x+V_{000}$, i.e. $\operatorname{Gr}(B, \bar{x})$ does not contain any non-zero vector in $V_{000}$. Then

$$
d(x)=c(\bar{x})+\sum_{A:|A| \geq 3}(\lceil|A| / 2\rceil-1)
$$

where $c(\bar{x})$ is the number of connected components of $\bar{x}$ and A runs through the set of maximal complete subgraphs of $\bar{x}$ and $|A|$ is the number of vertices in $A$.

2.2. Orbits of groups generated by symplectic transvections of a linearly independent subset which is not a basis

We first recall the following statement from [24].

Theorem 2.14 [24, Theorem 6.2] Let B be a linearly independent subset which is not a basis in $V$ and let $U$ denote the linear span of B. Suppose that $G r(B)$ is connected. If 
$G r(B)$ contains a subgraph which is equivalent to $E_{6}$, then $\Gamma_{B}$ has precisely two orbits in $(v+U)-V^{\Gamma_{B}}$, where $V^{\Gamma_{B}}$ is the set of vectors which are fixed by $\Gamma_{B}$. They are intersections of $(v+U)-V^{\Gamma_{B}}$ with the sets $Q_{B \cup\{v\}}^{-1}(0)$ and $Q_{B \cup\{v\}}^{-1}(1)$.

Our next result is a counterpart of Theorem 2.14 for graphs that do not contain $E_{6}$.

Theorem 2.15 Let B be a linearly independent subset which is not a basis in V. Suppose that $G r(B)$ is connected and does not contain a subgraph which is equivalent to $E_{6}$. Suppose also that $\operatorname{dim}(U) \geq 2$ where $U$ denotes the linear span of $B$. Then we have the following statements:

(i) If $\Omega\left(v, U_{000}\right) \neq\{0\}$, then there are precisely two $\Gamma_{B}$-orbits in $v+U$. They are intersections of $v+U$ with the sets $Q_{B \cup\{v\}}^{-1}(0)$ and $Q_{B \cup\{v\}}^{-1}(1)$.

(ii) If $\Omega\left(v, U_{000}\right)=\{0\}$ then $\Gamma_{B}$-orbits in $v+U$ are intersections of $v+U$ with the $\Gamma_{B \cup\{w\}}$ orbits in the linear span of $B \cup\{w\}$ for some $w \in v+U$ such that $\operatorname{Gr}(B \cup\{w\})$ does not contain any subgraph which is equivalent to $E_{6}$.

\subsection{Orbits of groups generated by non-symplectic transvections}

Let us first recall that, for an arbitrary bilinear form $\Omega$ on $V$ and a linearly independent set $B$, we define its graph $\operatorname{Gr}(B)$ as the graph whose vertex set is $B$ and $b_{i}, b_{j}$ in $B$ are connected if $\Omega\left(b_{i}, b_{j}\right)=1$ or $\Omega\left(b_{j}, b_{i}\right)=1$. The following result is a generalization of [8, Theorem 3$]$ :

Theorem 2.16 Suppose that $\Omega$ is a non-alternating bilinear form on the $\mathbb{F}_{2}$-space $V$. Let $B$ be a linearly independent subset whose graph is connected. Suppose that there exists a disjoint collection of connected graphs $B_{1}, \ldots, B_{r}$ with $B=\cup B_{i}$ such that $\left.\Omega\right|_{\mathbb{F}_{2}^{B_{i}}}$ is alternating and, for any $b_{i} \in B_{i}, b_{j} \in B_{j}$ with $i \neq j$, we have $\Omega\left(b_{i}, b_{j}\right)=1$ if and only if $j=i+1$. Suppose that $x \in V$ is not fixed by $\Gamma_{B}$ and let $L=\min \left\{j: x\right.$ is not fixed by $\left.\Gamma_{B_{j}}\right\}$. Then the $\Gamma_{B^{-}}$-orbit of $x$ coincides with the set $\Gamma_{B_{L}}\left(\pi_{L}(x)\right)+\mathbb{F}_{2}^{\cup>L}{ }_{j} B_{j}$ where $\Gamma_{B_{L}}\left(\pi_{L}(x)\right)$ is the $\Gamma_{B_{L}}$-orbit of the vector $\pi_{L}(x)$; here $\pi_{L}$ is the standard projection onto $\mathbb{F}_{2}^{B_{L}}$.

\section{Orbits of groups generated by symplectic transvections of a basis}

In this section, we prove Theorems 2.6-2.13. It will be convenient for us to prove, first, Theorem 2.8. Let us keep the notation of Section 2 and recall that $V_{000}$ is the set that consists of vectors $y$ in $V_{0}$ such that $y=x_{1}+x_{2}$ for some $x_{1}, x_{2} \in \Delta$. We also introduce the subspace $V_{00}=\left\{y \in V_{0}: Q_{B}(y)=0\right\}$ where $Q_{B}$ is the (unique) quadratic function associated with $B$ (c.f. Section 2). We note that $V_{000} \subset V_{00}$.

\subsection{Proof of Theorem 2.8}

To prove the theorem, it is enough to show that for any $x \in V-V_{0}$ and $u \in V_{000}$, the vectors $x$ and $x+u$ lie in the same $\Gamma_{B}$-orbit. Let us assume that $u=u_{1}+u_{2}$, where $u_{1}, u_{2} \in \Delta$. Since $B$ lies in $\Delta$, we may also assume, without loss of generality that, $u_{1} \in B$. We note that $\Omega\left(u_{1}, u_{2}\right)=0$ since $u_{1}+u_{2} \in V_{0}$. 
We claim that there exists $\gamma \in \Gamma_{B}$ such that $\Omega\left(\gamma(x), u_{1}\right)=1$. Suppose that $\Omega\left(\gamma(x), u_{1}\right)=$ 0 for all $\gamma \in \Gamma_{B}$. Then $\Omega\left(x, \gamma\left(u_{1}\right)\right)=0$ for all $\gamma \in \Gamma_{B}$. This implies, in particular, that $\Omega(x, b)=0$ for all $b \in B$, because $u_{1} \in B$ lies in the orbit $\Delta$. This would imply that $x \in V_{0}$, resulting in a contradiction because $x \notin V_{0}$. We note that $\Omega\left(\gamma(x), u_{2}\right)=1$ because $u=u_{1}+u_{2} \in V_{0}$.

Let us now consider $\gamma^{-1} \tau_{u_{2}} \tau_{u_{1}} \gamma$. This automorphism is in $\Gamma_{B}$ because $\tau_{u_{2}} \in \Gamma_{B}$ by [1, Proposition 3.1]. Then $\gamma^{-1} \tau_{u_{2}} \tau_{u_{1}} \gamma(x)=x+u_{1}+u_{2}=x+u$, hence $x$ and $x+u$ are contained in the same $\Gamma_{B}$-orbit.

We obtain the remaining part of Theorem 2.8 from the following explicit description of $V_{0}, V_{00}, V_{000}$.

Proposition 3.1 The spaces $V_{0}, V_{00}$ and $V_{000}$ have the following descriptions in terms of the trees in figures $2-5$.

(i) Suppose that $B$ is of type $D_{m, k}$ with $m \geq 2, k \geq 1$ indexed as in figure 2.

If $m$ is odd, then $V_{0}=V_{00}=V_{000}=$ linear span of $\left\{c_{1}+c_{2}, c_{1}+c_{3}, \ldots, c_{1}+c_{k}\right\}$.

If $m=2$, then $V_{0}=V_{00}=V_{000}=$ linear span of $\left\{a_{1}+c_{1}, c_{1}+c_{2}, c_{1}+c_{3}, \ldots, c_{1}+\right.$ $\left.c_{k}\right\}$. If $m>2$ and $m \equiv 2 \bmod (4)$, then $V_{000}=$ linear span of $\left\{c_{1}+c_{2}, c_{1}+c_{3}, \ldots, c_{1}+\right.$ $\left.c_{k}\right\}$ and $V_{0}=V_{00}=$ linear span of $V_{000} \cup\left\{a_{1}+a_{3}+\cdots+a_{m-1}+c_{1}\right\}$.

If $m>2$ and $m \equiv 0 \bmod (4)$, then $V_{00}=V_{000}=$ linear span of $\left\{c_{1}+c_{2}, c_{1}+\right.$ $\left.c_{3}, \ldots, c_{1}+c_{k}\right\}$ and $V_{0}=$ linear span of $\left(V_{000} \cup\left\{a_{1}+a_{3}+\cdots+a_{m-1}+c_{1}\right\}\right)$.

(ii) If $B$ is as in figure 3 or figure 4 , then $V_{0}=V_{00}=V_{000}=$ linear span of $\left\{c_{1}+c_{2}, c_{1}+\right.$ $\left.c_{3}, \ldots, c_{1}+c_{k}\right\}$

(iii) If $B$ is as in figure 5, then $V_{00}=V_{000}=$ linear span of $\left\{c_{1}+c_{2}, c_{1}+c_{3}, \ldots, c_{1}+c_{k}\right\}$ and $V_{0}=$ linear span of $V_{000} \cup\left\{a_{1}+a_{2}+a_{4}\right\}$.

Corollary 3.2 For any basis $B$ whose graph is equivalent to a tree of type $D_{m, k}$ with $m \geq 2, k \geq 1$, we have the following:

(i) If $m>2$, then $\operatorname{dim}\left(V_{000}\right)=k-1$. If $m=2$, then $\operatorname{dim}\left(V_{0}\right)=\operatorname{dim}\left(V_{000}\right)=k$.

(ii) The number of fixed points of $\Gamma_{B}$ in $V$ is $2^{k-1}$ if $m$ is odd, and $2^{k}$ if $m$ is even.

(iii) The number of $\Gamma_{B}$-orbits which are not fixed points is $(m+1) / 2$ if $m$ is odd, and $m / 2$ if $m$ is even.

We record the following trivial statement for later use.

Proposition 3.3 Suppose that $C=\left[c_{1}, c_{2}, \ldots, c_{k}\right], k \geq 3$, is a linearly independent subset whose graph is a cycle. Let $W$ be the subspace spanned by $C$. Then the vector $c_{1}+c_{2}+\cdots+c_{k} \in W_{000}$. If $k=4$, then, for any $c_{i}$ and $c_{j}$ which are not connected to each other (in $\operatorname{Gr}(C))$, we have $c_{i}+c_{j} \in W_{000}$.

\subsection{Proof of Theorem 2.6}

Let us first introduce some terminology. For a vector $x \in V$, we say that $x=x_{1}+\cdots+x_{s}$ is a $\Delta$-decomposition of $x$ if $x_{1}, \ldots, x_{s} \in \Delta$ and $\Omega\left(x_{i}, x_{j}\right)=0$ for all $i, j=1, \ldots, s$, 
here $\Delta$ denotes the $\Gamma_{B}$-orbit that contains $B$ (and any basis equivalent to it). If $Q_{B}(x)=1$ (resp. $Q_{B}(x)=0$ ), then any $\Delta$-decomposition of $x$ has an odd (resp. even) number of components. Let us also note that

$$
d(x)=\min \left\{s: x \text { has a } \Delta \text {-decomposition } x=x_{1}+\cdots+x_{s}\right\} .
$$

We first prove the existence of a $\Delta$-decomposition for an arbitrary $x \in V-V_{0}$. Since any connected graph is equivalent to a tree, we may assume that $B$ is a tree. Let $B_{1}, \ldots, B_{s}$ be the connected components of $\operatorname{Gr}(B, x)$ and let $x_{1}, \ldots, x_{s}$ be the corresponding vectors in $V$. We claim that $x=x_{1}+\cdots+x_{s}$ is a $\Delta$-decomposition for $x$. We note that $\Omega\left(x_{i}, x_{j}\right)=0$ for all $i \neq j$. To show that $x_{i} \in \Delta$ for $i=1, \ldots, s$, it is enough to show, without loss of generality, that $x_{1} \in \Delta$. Let us assume $x_{1}=b_{1}+\cdots+b_{k}, b_{i} \in B$. Since $\operatorname{Gr}\left(B, x_{1}\right)$ is a tree, it has a leaf, i.e. a vertex, say $b_{k}$, which is connected to precisely one vertex in $\operatorname{Gr}(B, x)$. Then $\operatorname{Gr}\left(B, \tau_{b_{k}}(x)\right)$ is a tree with $k-1$ vertices. By induction, we obtain $\gamma \in \Gamma_{B}$ such that $\gamma\left(x_{1}\right) \in B$, i.e. $x_{1} \in \Delta$.

Let us also note that, for any $\Delta$-decomposition $x=x_{1}+\cdots+x_{s}$ and any $\gamma \in \Gamma$, we have a $\Delta$-decomposition $\gamma(x)=\gamma\left(x_{1}\right)+\cdots+\gamma\left(x_{s}\right)$. Thus, we have

$$
d(x)=d(\gamma(x)) \text { for any } \gamma \in \Gamma \text {. }
$$

Lemma 3.4 Suppose that $B$ is equivalent to a tree that contains $E_{6}$. Then, for any $x \in$ $V-V_{0}$, we have the following:

(i) $d(x)=1$ if and only if $Q_{B}(x)=1$

(ii) $d(x)=2$ if and only if $Q_{B}(x)=0$

The "only if" parts are clear. To prove the "if" parts, let us first assume $Q_{B}(x)=1$. Then $x \in \Delta$, by Theorem 2.3, hence $d(x)=1$. Let us now assume that $Q_{B}(x)=0$ and let $x=x_{1}+\cdots+x_{2 l}$, be a $\Delta$-decomposition. Then there exist $j \in\{1, \ldots, 2 l\}$ such that $x+x_{j} \notin V_{0}$ (otherwise $x=\sum\left(x+x_{j}\right) \in V_{0}$ ). Since $x=\left(x+x_{j}\right)+x_{j}$, and $Q_{B}\left(x+x_{j}\right)=Q_{B}\left(x_{j}\right)=1$, we have $d(x)=2$ and we are done.

To complete the proof of the theorem let us now assume that $B$ is equivalent to a tree $B^{\prime}$ which is of type $D_{m, k}$. We claim that, for any $x \in V-V_{0}$, the number $d(x)$ is equal to the number of connected components of $\operatorname{Gr}\left(B^{\prime}, p(x)\right)$, where $p$ is the function defined in Theorem 2.5. In view of Theorem 2.8 and (3.1), this claim follows from the following stronger statement.

Lemma 3.5 Let $B$ be a basis equivalent to a tree $B^{\prime}$ of type $D_{m, k}$ with $m \geq 2$ and $k \geq 1$. Suppose that $x \in V$ and let $x=x_{1}+x_{2} \cdots+x_{d}$ be a $\Delta$-decomposition, i.e. $x_{1}, \ldots, x_{s} \in \Delta$ and $\Omega\left(x_{i}, x_{j}\right)=0$ for all $i, j=1, \ldots, d$. Suppose also that

$$
\text { for any } i \neq j, \text { we have } x_{i}+x_{j} \notin V_{000} \text {. }
$$

Then there exists $\alpha \in \Gamma_{B}$ such that $\alpha(x)=y_{1}+y_{2}+\cdots+y_{d}$, where $y_{i}=\alpha\left(x_{i}\right) \in B^{\prime}$. 
Let us assume that $B^{\prime}$ is indexed as in figure 2. Applying if necessary an element of $\Gamma_{B}$, we may assume that $x_{1}=a_{1}$. By our assumption in (3.2), all $x_{i}$ for $i \geq 2$ are contained in $\left\{a_{3}, \ldots, a_{m}, c_{i}\right\}$ (Theorem 2.5, Proposition 3.1). Our claim follows by induction on $d$. This completes the proof of Theorem 2.6.

\subsection{Proof of Theorem 2.7}

We will first prove that

a graph $B$ is equivalent to a tree of type $D_{m, 1}$ if and only if it does not have subgraphs of the form in (2.1)

Let us denote by $F$ the set that consists of the graphs in (2.1). Since $B$ is equivalent to a tree $T$ of type $D_{m, 1}$, there exist a sequence of basic moves $\phi_{c_{r}, a_{r}}, \ldots, \phi_{c_{2}, a_{2}}, \phi_{c_{1}, a_{1}}$ such that $B=\phi_{c_{r}, a_{r}} \circ \cdots \circ \phi_{c_{2}, a_{2}} \circ \phi_{c_{1}, a_{1}}(T)$. We will show by induction on $r$ that each graph $B_{i}=\phi_{c_{i}, a_{i}} \ldots \phi_{c_{2}, a_{2}} \phi_{c_{1}, a_{1}}(T), 1 \leq i \leq r$, in particular $B=B_{r}$, contains a subgraph that belongs to $F$. The basis of the induction is the fact that any tree of type $D_{m, 1}$ does not contain any subgraph that belongs to $F$. The induction follows from the following lemma:

Lemma 3.6 If a graph $G$ contains a subgraph $X \in F$, then, for any basic move $\phi_{c, a}$, the graph $\phi_{c, a}(G)$ contains a subgraph $X^{\prime}$ which belongs to $F$.

Since the basic move exchanges $c$ by $c+a$ and fixes the other elements of $G$, we may assume that $c$ is in $X$. It follows from a direct check that

if $a \in X$, then $\phi_{c, a}(G)$ contains a subgraph $X^{\prime}$ which belongs to $F$.

If $a \notin X$ and it is not connected to any vertex $v$ in $X$ such that $v \neq c$, then the graph $\phi_{c, a}(X)=X$ is in $F$. Let us now assume that $a \notin X$ and it is connected to a vertex $v \neq c$ in $X$. By (3.4), we may also assume that $a$ is not contained in any subgraph which is in $F$. Then the subgraph $X a$ with the vertices $X \cup\{a\}$ is of the form in figure 6 as could be verified easily. We note that the graph $X^{\prime}=\phi_{c, a}(X a)$ belongs to $F$.

We complete the proof of Theorem 2.7 by the following lemma.

Lemma 3.7 Let $G$ be a connected graph that contains a subgraph $X$ which is equivalent to $E_{6}$. Then, for any a, $c$ in $G$ such that $\Omega(a, c)=1$, the graph $\phi_{c, a}(G)$ contains a subgraph $X^{\prime}$ which is equivalent to $E_{6}$.

If $c \notin X$, then we may take $X^{\prime}=X$. If $c \in X$ and $a \in X$, then we may take $X^{\prime}=\phi_{c, a}(X)$. Let us now assume that $c \in X$ and $a \notin X$. We write $X a$ to denote the (connected) graph with the vertices in $X \cup\{a\}$ and denote by $U$ the linear span of $X a$. We will show that

the graph $\phi_{c, a}(X a)$ contains a subgraph $X^{\prime}$ which is equivalent to $E_{6}$. 


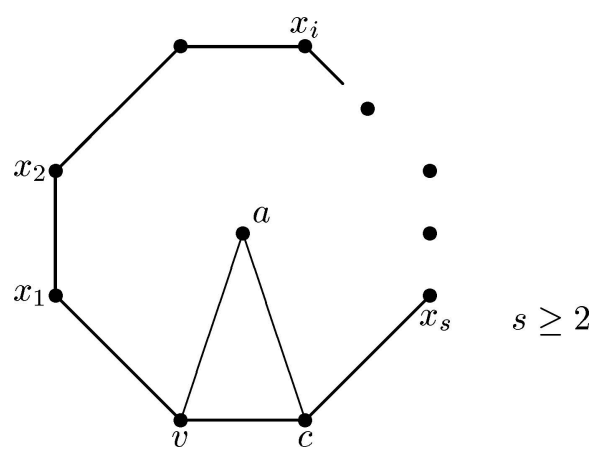

Figure 6. The vertex $a$ is connected to the cycle without being contained in a subgraph which is of the form in (2.1).

By Theorem 2.3, the graph $X c$ is equivalent to a tree of the form in figure 3 or figure 5 . Let us first assume that $X a$, hence $Y=\phi_{c, a}(X a)$, is equivalent to a tree of the form in figure 3. Then $\operatorname{dim}\left(U_{00}\right)=1$ by Proposition 3.1. Let $y$ in $U_{00}$ be such that $y \neq 0$, and let $b$ be a vertex in $\operatorname{Gr}(Y, y)$ such that $X^{\prime}=Y-\{y\}$ is connected. Then $\operatorname{Arf}\left(Q_{X^{\prime}}\right)=1$, so $X^{\prime}$ is equivalent to $E_{6}$ by Theorem 2.3. Let us now assume that $X a$ is equivalent to a tree of the form in figure 5 . We will show, using a case by case analysis, that the graph $Y=\phi_{c, a}(X a)$ contains a subgraph $X^{\prime}$ which is equivalent to $E_{6}$, and this will complete the proof of Lemma 3.7.

For the remaining part of the proof, we assume that $Y=\left\{b_{1}, b_{2}, \ldots, b_{6}, b_{7}\right\}$. We denote by $U$ the linear span of $Y$. We note that $\operatorname{dim}\left(U_{0}\right)=1$ and $\operatorname{dim}\left(U_{00}\right)=\operatorname{dim}\left(U_{000}\right)=0$. We also recall that we always assume a subgraph to be an induced subgraph.

Case $1 Y$ is a cycle of length 7.

Any cycle $C$ of length $r \geq 4$ is equivalent to a tree of type $D_{r-2,2}$. Since $X a$, hence $Y$, is equivalent to a tree that contains $E_{6}$, this case is not possible (Proposition 2.4).

Case $2 Y$ contains a cycle $C$ of length 6 .

Let us assume without loss of generality that $C=\left[b_{1}, b_{2}, \ldots, b_{6}\right]$. Since $\operatorname{dim}\left(U_{00}\right)=$ 0 , the vertex $b_{7}$ is connected to an odd number of vertices in $C$ (otherwise the vector $b_{1}+b_{2}+\cdots+b_{6}$ is in $U_{00}$, c.f. Proposition 3.3). Thus $Y$ is one of the graphs in figures 7-9. We note that each of the graphs in figures 7-9 contains a subgraph equivalent to $E_{6}$ which is marked by thick lines.

Case $3 Y$ contains a cycle $C$ of length 5 .

Let us assume without loss of generality that $C=\left[b_{1}, b_{2}, \ldots, b_{5}\right]$. Since $\operatorname{dim}\left(U_{00}\right)=0$, there is a vertex, say $b_{6}$, in $Y$ that is connected to an odd number of vertices in $C$ (otherwise 

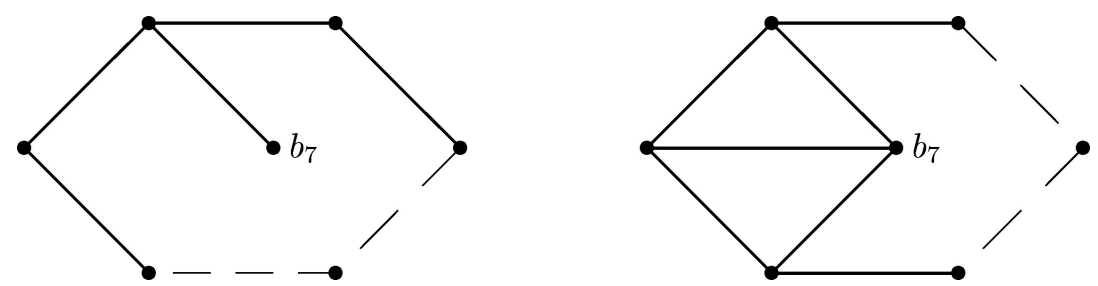

Figure 7. The vertex $b_{7}$ is connected to an odd number of vertices in the cycle of length 6 , forming a subgraph equivalent to $E_{6}$.
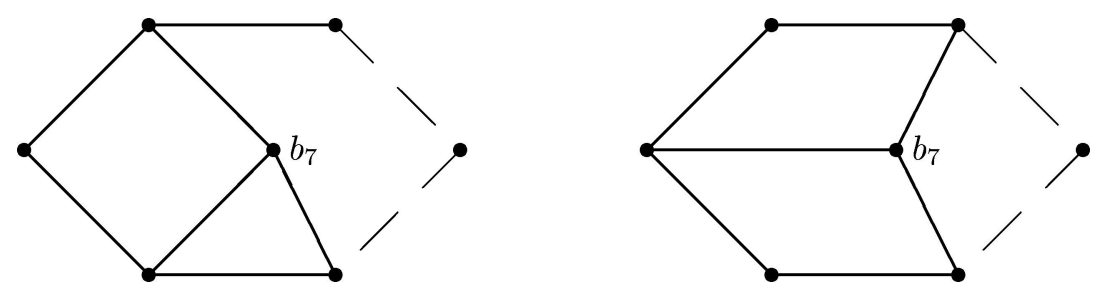

Figure 8 . The vertex $b_{7}$ is connected to 3 vertices in the cycle of length 6 , forming a subgraph equivalent to $E_{6}$.

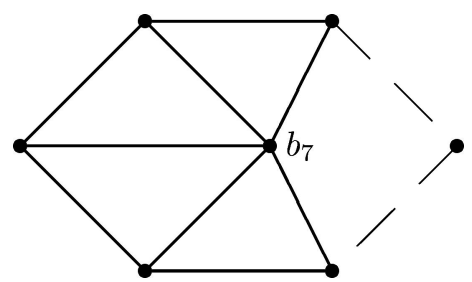

Figure 9. The vertex $b_{7}$ is connected to 5 vertices in the cycle of length 6 , forming a subgraph equivalent to $E_{6}$.

the vector $b_{1}+b_{2}+\cdots+b_{5}$ is in $\left.U_{00}\right)$. Then the subgraph with the vertices $\left\{b_{1}, b_{2}, \ldots, b_{6}\right\}$ is one of the graphs in figure 10 or figure 11 . We note that each of the graphs in figures 10 and 11 is equivalent to $E_{6}$.

Case $4 Y$ contains a cycle $C$ of length 4 .

Let us assume, without loss of generality, that $C=\left[b_{1}, b_{2}, b_{3}, b_{4}\right]$. We note there is a vertex which is not in $C$ but connected to an odd number of vertices in $C$ (Proposition 3.3).

Subcase 4.1 There is a vertex $v \notin C$ such that $v$ is connected to precisely 3 vertices in $C$.

Let us assume, without loss of generality, that $v$ is connected to the vertices $b_{1}, b_{2}, b_{3}$. Since $\operatorname{dim}\left(U_{00}\right)=0$, there is a vertex $v^{\prime} \neq v$ such that $v^{\prime} \notin C$ and connected to precisely one vertex, say $b_{1}$, in $\left\{b_{1}, b_{3}\right\}$ (otherwise $b_{1}+b_{3}$ in $U_{00}$ ). 

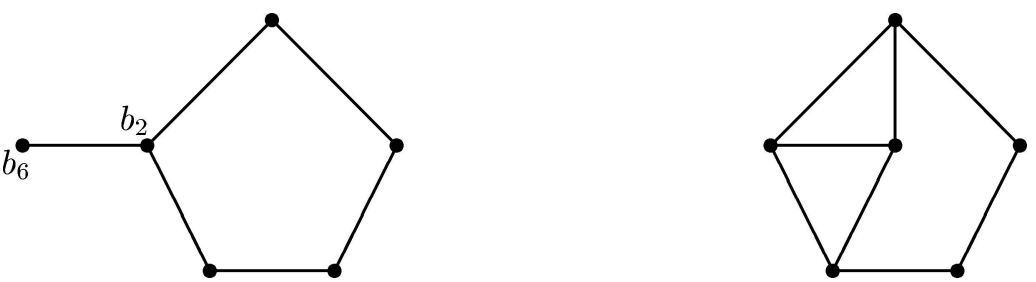

Figure 10. Each graph is equivalent to $E_{6}$.
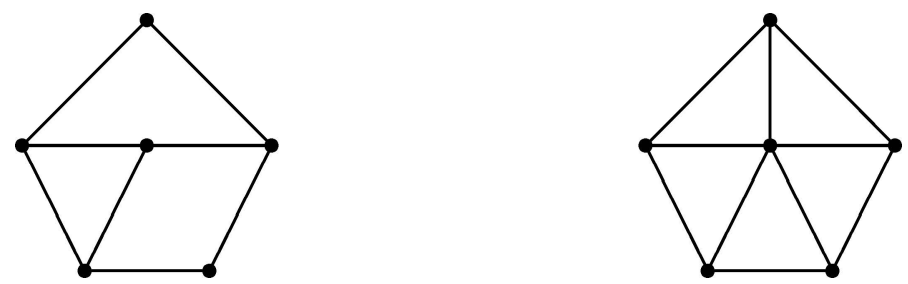

Figure 11. Each graph is equivalent to $E_{6}$
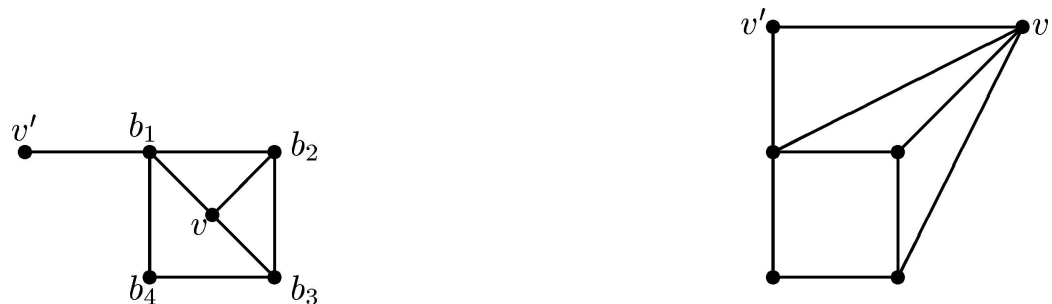

Figure 12. Each graph is equivalent to $E_{6}$

Subsubcase 4.1.1 $v^{\prime}$ is not connected to any vertex in $\left\{b_{2}, b_{4}\right\}$. Then the subgraph $X^{\prime}$ with the vertices $C \cup\left\{v, v^{\prime}\right\}$ is one of the graphs in figure 12 and it is equivalent to $E_{6}$.

Subsubcase 4.1.2 $v^{\prime}$ is connected to precisely one vertex in $\left\{b_{2}, b_{4}\right\}$.

Let $X^{\prime}$ be the subgraph with the vertices $C \cup\left\{v, v^{\prime}\right\}$. Then we have the following:

(i) If $v^{\prime}$ is not connected to $v$, then the graph $X^{\prime}$ is one of the graphs in figure 13 which are equivalent to $E_{6}$.

(ii) If $v^{\prime}$ is connected to $v$, then (i) applies to $\phi_{v^{\prime}, b_{1}}\left(X^{\prime}\right)$, thus $X^{\prime}$ is equivalent to $E_{6}$. 


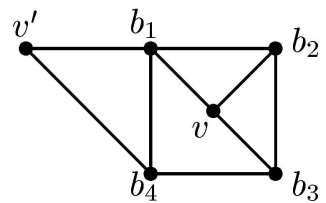

Figure 13. Each graph is equivalent to $E_{6}$.

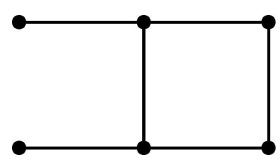

Figure 14. Each graph is equivalent to $E_{6}$
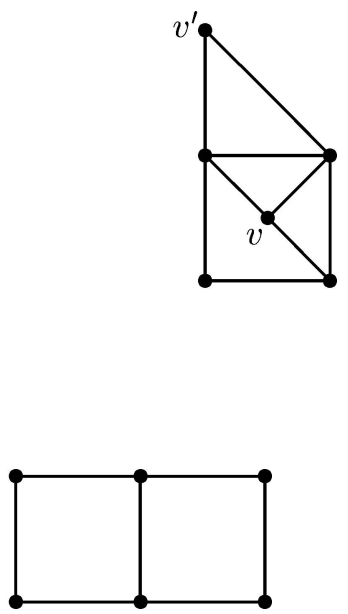

Subsubcase 4.1.3 $v^{\prime}$ is connected to precisely two vertices in $\left\{b_{2}, b_{4}\right\}$. Let $X^{\prime}$ be the subgraph with the vertices $C \cup\left\{v, v^{\prime}\right\}$. Then Subcase 4.1.1 applies to $\phi_{v^{\prime}, b_{1}}\left(X^{\prime}\right)$, so $X^{\prime}$ is equivalent to $E_{6}$.

Subcase 4.2 Subcase 4.1. does not hold. Since $\operatorname{dim}\left(U_{00}\right)=0$, there are vertices $v, v^{\prime} \notin C$ which are connected to adjacent vertices in $C$. Then the graph $X^{\prime}$ with the vertices $C \cup\left\{v, v^{\prime}\right\}$ is one of the graphs in figure 14 which are equivalent to $E_{6}$.

Case $5 Y$ contains two adjacent triangles sharing precisely one common edge.

Let $T=\left\{b_{1}, b_{2}, b_{3}, b_{4}\right\}$ be the subgraph formed by the adjacent triangles sharing the common edge $\left[b_{2}, b_{3}\right]$. We note that the graph $T^{\prime}=\phi_{b_{1}, b_{2}}(T)$ is a cycle of length 4 . By our analysis in Case 4, the graph $T^{\prime}$ is contained in a graph $E$ which is equivalent to $E_{6}$. Then $T$ is contained in $X^{\prime}=\phi_{b_{1}, b_{2}}(E)$.

Case 6 Y contains a subgraph $D$ of the form $D_{2,2}$.

Let $D=\left\{a_{1}, a_{2}, c_{1}, c_{2}\right\}$ be indexed as in figure 2. We note that the graph $D^{\prime}=\phi_{a_{1}, a_{2}}(D)$ is formed by two adjacent triangles. By our analysis in Case 5 , the graph $D^{\prime}$ is contained in a graph $E$ which is equivalent to $E_{6}$. Then $D$ is contained in $X^{\prime}=\phi_{a_{1}, a_{2}}(E)$.

Case 7 None of the above cases happen.

Then $Y$ is equivalent to a tree of type $D_{m, 1}$ by (3.3), which contradicts to our assumption that $Y$ is equivalent to a tree that contains $E_{6}$ (Proposition 2.4). This completes the proof of Theorem 2.7. 


\subsection{Proof of Theorem 2.9}

Let us first assume that $X$ has precisely 4 vertices. Then $X$ is equivalent to a cycle $X^{\prime}=$ $\left[c_{1}, c_{2}, c_{3}, c_{4}\right]$ (such that $\Omega\left(c_{1}, c_{3}\right)=\Omega\left(c_{2}, c_{4}\right)=0$ ). We claim that

$$
\text { there is } x \in\left\{c_{1}+c_{2}+c_{3}+c_{4}, c_{1}+c_{3}, c_{2}+c_{4}\right\} \text { such that } x \in V_{000}
$$

Suppose that (3.6) is not satisfied. Then there is a vector $v \notin X^{\prime}$ such that $\Omega\left(v, c_{1}+c_{2}+\right.$ $\left.c_{3}+c_{4}\right)=1$. We may assume, without loss of generality, that $\Omega\left(v, c_{2}+c_{4}\right)=0$. Then there is also a vector $v^{\prime} \notin X^{\prime}$ such that $\Omega\left(v^{\prime}, c_{2}+c_{4}\right)=1$ (because we assume that (3.6) is not satisfied). Then the subgraph with the vertices $X^{\prime} \cup\left\{v, v^{\prime}\right\}$ is equivalent to $E_{6}$ (by our analysis in Case 4 of the proof of Lemma 3.7). This contradicts Proposition 2.4.

Let us now assume that $X$ has at least 5 vertices, i.e. $X=\left[c_{1}, \ldots, c_{r}\right]$ is a cycle whose length $r$ is greater than or equal to 5 . We claim that $x=c_{1}+c_{2}+\cdots+c_{r}$ is in $V_{000}$. Let $B^{\prime}=\phi_{c_{r-1}, c_{r}}(B)$. Then $\operatorname{Gr}\left(B^{\prime}, x\right)$ is a cycle of length $r-1$. Then the claim follows by induction on $r$. (Here we note the following: if $r=5$ then $\operatorname{Gr}\left(B^{\prime}, x\right)=\left[c_{1}, c_{2}, c_{3}, c_{4}+c_{5}\right]$ is a cycle of length 4 such that $c_{1}+c_{3}, c_{2}+c_{4}+c_{5} \notin V_{000}$ because $\Omega\left(c_{1}+c_{3}, c_{5}\right)=$ $1, \Omega\left(c_{2}+c_{4}+c_{5}, c_{5}\right)=1$.)

The remaining part of Theorem 2.9 follows from Lemma 3.5.

\subsection{Proof of Theorem 2.10}

We will show that, for any $B$ which is equivalent to a tree that contains $E_{6}$, there is a subgraph $X$ of the form in (2.1) such that $\mathbb{F}_{2}^{X} \cap V_{000}=\{0\}$. For such a $B$, there is a subgraph $E \subset B$ which is equivalent to $E_{6}$ (Theorem 2.7). Let us denote by $U$ the linear span of vectors contained in $E$. Then $\operatorname{dim}\left(U_{0}\right)=0$ by Proposition 3.1. Since $E$ is not equivalent to any tree of type $D_{m, 1}$ (Proposition 2.4), it contains a subgraph $X$ such that $X$ is of the form in (3.1) (Theorem 2.7). Then $\mathbb{F}_{2}^{X} \cap V_{000}=\{0\}$ because $\operatorname{dim}\left(U_{0}\right)=0$ and $\mathbb{F}_{2}^{X} \cap V_{000} \subset U_{0}$. This completes the proof of the theorem.

\subsection{Proof of Theorem 2.11}

For any basis $B$ which is equivalent to a tree of type $D_{m, k}$ we denote by $B_{000}$ the set that consists of vectors $x \in V_{000}$ such that $\operatorname{Gr}(B, x)$ is contained in a subgraph of the form in (3.1). Then Theorem 2.11 is equivalent to the following statement:

for any basis $B$ which is equivalent to a tree of type $D_{m, k}$, the set $B_{000}$ spans $V_{000}$.

Let us first note that (3.7) holds for $B$ which is a tree of type $D_{m, k}$ (Proposition 3.1). Thus, to prove (3.7), it is enough to prove the following statement:

if $x$ in $B_{000}$, then, for any basis $B^{\prime}=\phi_{c, a}(B)$, the vector $x$ is in the linear span of $B_{000}^{\prime}$. 
It follows from a direct check that

if $a, c$ are in $\operatorname{Gr}(B, x)$, then (3.8) is satisfied.

Let us now assume, without loss of generality, that $c \in \operatorname{Gr}(B, x)$, and $a \notin G r(B, x)$. We will establish (3.8) using a case by case analysis.

Case $1 \quad G r(B, x)$ has precisely two vertices.

In this case $\operatorname{Gr}(B, x)$ is contained in a subgraph $X$ which is a tree of type $D_{2,2}$ or a cycle of length 4 . Let $x=c+b$ be the expansion of $x$ in the basis $B$. We note that $a$ is connected to both $c$ and $b$ because $x \in V_{0}$. Then $\operatorname{Gr}\left(B^{\prime}, x\right)$ is the triangle with the vertices $\{c+a, a, b\}$ and it is in $B_{000}^{\prime}$.

Case $2 G r(B, x)$ has precisely three vertices

We note that in this case $\operatorname{Gr}(B, x)$ is a triangle. Let $x=c+b_{1}+b_{2}$ be the expansion of $x$ in the basis $B$. Then $\operatorname{Gr}\left(B^{\prime}, x\right)$ is the cycle with vertices $\left\{c+a, b_{1}, b_{2}, a\right\}$. In particular it is in $B_{000}^{\prime}$.

Case $3 \operatorname{Gr}(B, x)$ has precisely 4 vertices

We note that in this case $\operatorname{Gr}(B, x)$ is a cycle of length 4 . Let us assume that $x=$ $c+b_{1}+b_{2}+b_{3}$ is the expansion of $x$ in the basis $B$.

Subcase 3.1 a is connected to precisely one vertex, say $b$, in $\left\{b_{1}, b_{2}, b_{3}\right\}$. If $b$ is connected to $c$, then $\operatorname{Gr}\left(B^{\prime}, x\right)$ is a cycle of length of 5 so $x \in B_{000}^{\prime}$. Let us now assume that $b$ is not connected to $c$. We may also assume that $b=b_{2}$ (see figure 15(i)). Then the vectors $x_{1}=b_{1}+a$ and $x_{2}=b_{2}+b_{3}+c+a$ are in $V_{000}$ by Theorem 2.9. They are also in the linear span of $B_{000}^{\prime}$ by (3.9), so is $x=x_{1}+x_{2}$.

Subcase $3.2 a$ is connected to all of the vertices in $\left\{b_{1}, b_{2}, b_{3}\right\}$. In this case, the vectors $x_{1}=c+a+b_{1}$ and $x_{2}=b_{2}+a+b_{3}$ are in $V_{000}$ by Theorem 2.9 (see figure 15.(ii)). They are also in the linear span of $B_{000}^{\prime}$ by (3.9), so is $x=x_{1}+x_{2}$.

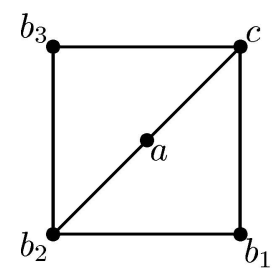

(i)

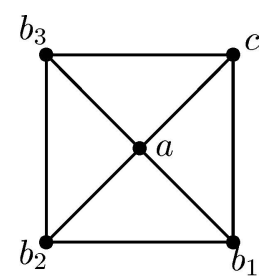

(ii)

Figure 15. The vector $x=b_{1}+b_{2}+b_{3}+c$ is in $V_{000}$. 


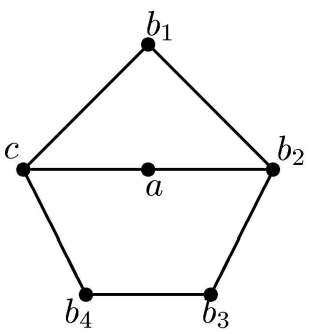

(i)

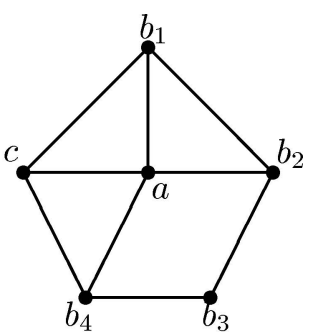

(ii)

Figure 16. The vector $x=b_{1}+b_{2}+b_{3}+b_{4}+c$ is in $V_{000}$.

Case $4 \operatorname{Gr}(B, x)$ contains precisely 5 vertices.

In this case $\operatorname{Gr}(B, x)=\left[c, b_{1}, b_{2}, b_{3}, b_{4}\right]$ is a cycle of length 5 .

Subcase 4.1 a is connected to precisely one vertex, say $b$, in $\left\{b_{1}, b_{2}, b_{3}, b_{4}\right\}$.

If $b$ is connected to $c$, then $\operatorname{Gr}\left(B^{\prime}, x\right)$ is a cycle of length 5, so $x \in B_{000}^{\prime}$. Let us now assume that $b$ is not connected to $c$. We may also assume, without loss of generality, that $b=b_{2}$ (see figure 16(i)). Then the vectors $x_{1}=b_{1}+a, x_{2}=c+a+b_{2}+b_{3}+b_{4}$ are in $V_{000}$ by Theorem 2.9. They are also in the linear span of $B_{000}^{\prime}$ by (3.9), so is $x=x_{1}+x_{2}$.

Subcase 4.2 a is connected to precisely three vertices in $\left\{b_{1}, b_{2}, b_{3}, b_{4}\right\}$.

In this case, we may assume that $\operatorname{Gr}(B, x)$ is as in figure 16(ii). We note that $x_{1}=$ $c+b_{1}+a$ and $x_{2}=a=b_{2}+b_{3}+b_{4}$ are in $V_{000}$. They are also in the linear span of $B_{000}^{\prime}$ by (3.9), so is $x=x_{1}+x_{2}$.

Case $5 \operatorname{Gr}(B, x)$ has at least 6 vertices.

We note that the graph $\operatorname{Gr}(B, x)=\left[c, b_{1}, \ldots, b_{r}\right]$ is a cycle of length $r, r \geq 6$.

Subcase $5.1 a$ is connected to precisely one vertex, say $b$ in $b_{1}, b_{2}, b_{3}, b_{4}, b_{5}$.

If $b$ is connected to $c$, then $\operatorname{Gr}\left(B^{\prime}, x\right)$ is a cycle of length of $r+1$, so $x \in B_{000}^{\prime}$ Let us now assume that $b$ is not connected to $c$ and denote the graph with the vertices $\operatorname{Gr}(B, x) \cup\{a\}$ by $X a$. One could easily check the following: if $X a$ does not contain a cycle of length 4 then it contains a subgraph which is $E_{6}$, contradicting to our assumption that $B$ does not contain a subgraph equivalent to $E_{6}$. If $X a$ contains a cycle of length 4 , then it is of the form in figure 17. By the same arguments as in Case 5, one could show that $x$ is in the linear span of $B_{000}^{\prime}$.

Subcase 5.2 Let us now assume that $a$ is connected to (an odd number) $k$ vertices in $b_{1}, b_{2}, \ldots, b_{r}$ with $k \geq 1$. One could easily check that $X a$ contains a subgraph equivalent to $E_{6}$ unless it is of the form in figure 18. By the same arguments as in Case 5, one could show that $x$ is in the linear span of $B_{000}^{\prime}$. 


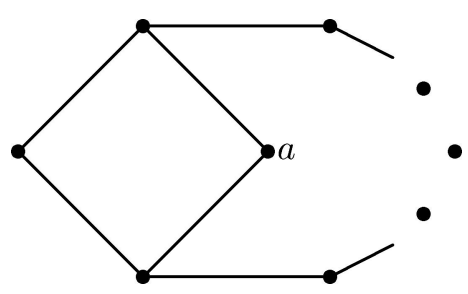

Figure 17. The graph does not contain any subgraph equivalent to $E_{6}$.

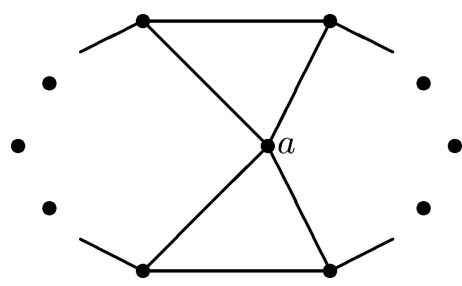

Figure 18. The graph does not contain any subgraph equivalent to $E_{6}$.

\subsection{Proof of Theorem 2.13}

Let us first introduce some notation. For any vector $v \in V$, we denote by $C(B, v)$ the set of connected components of $\operatorname{Gr}(B, v)$. We denote by $M(B, v)$ the set of maximal complete subgraphs (of $\operatorname{Gr}(B, v)$ ) with at least three vertices. By some abuse of notation, we will also use $\bar{x}$ to denote the vector that corresponds to the graph $\bar{x}$. We will denote by $d_{B}(\bar{x})$ the expression on the right side of (2.2), thus we will show that $d(x)=d_{B}(\bar{x})$ for any $x \in V-V_{0}$ and any $B$ which is equivalent to a tree of type $D_{m, k}$.

Since $\bar{x}$ is minimal, each connected component of $\operatorname{Gr}(B, \bar{x})$ is equivalent to a tree of type $D_{m, 1}$ (Theorems 2.11,2.7). Let $A=\left\{a_{1}, \ldots, a_{r}\right\}, r \geq 3$ be a maximal complete subgraph which is contained in a connected component $C$ of $\bar{x}$. For any vertex $b$ in $C$ such that $b$ is not in $A$, we denote by $A(b)$ the vertex in $A$ which is closest to $b$ (such a vertex exists by (2.1)). For any vertex $a \in A$, we define $H(a)=\{b \in C-A: A(b)=a\} \cup\{a\}$ and for a pair $\left\{a_{i}, a_{j}\right\}$ of vertices in $A$, we define $H\left(a_{i}+a_{j}\right)=\left\{b \in C-A: A(b)=a_{i}\right.$ or $\left.A(b)=a_{j}\right\} \cup\left\{a_{i}+a_{j}\right\}$. If $r$ is even (resp. odd) let

$$
B^{\prime}=\phi_{a_{r-1}, a_{r}} \circ \cdots \circ \phi_{a_{3}, a_{4}} \circ \phi_{a_{1}, a_{2}}\left(\text { resp. } B^{\prime}=\phi_{a_{r-2}, a_{r-1}} \circ \cdots \circ \phi_{a_{3}, a_{4}} \circ \phi_{a_{1}, a_{2}}\right) .
$$

Then $C\left(B^{\prime}, \bar{x}\right)=(C(B, \bar{x})-C) \cup\left\{H\left(a_{1}+a_{2}\right), H\left(a_{3}+a_{4}\right), \ldots, H\left(a_{r-1}+a_{r}\right)\right\}$ (resp. $\left.C\left(B^{\prime}, \bar{x}\right)=(C(B, \bar{x})-C) \cup\left\{H\left(a_{1}+a_{2}\right), H\left(a_{3}+a_{4}\right), \ldots, H\left(a_{r-2}+a_{r-1}\right)\right\}, H\left(a_{r}\right)\right)$, and $M\left(B^{\prime}, x\right)=M(B, x)-A$. We note that $d_{B}(\bar{x})=d_{B^{\prime}}(\bar{x})$ and $\bar{x}$ is minimal. Continuing this procedure, we obtain a basis $B^{\prime \prime}$ which is equivalent $B$ such that each connected component of $\operatorname{Gr}\left(B^{\prime \prime}, \bar{x}\right)$ is a chain. Then $d_{B}(\bar{x})=d_{B^{\prime \prime}}(\bar{x})$ is equal to the number of connected components of $\operatorname{Gr}\left(B^{\prime \prime}, \bar{x}\right)$. (Note that $\bar{x}$ is also minimal with respect to $B^{\prime}$ ). Since each connected component of $\operatorname{Gr}\left(B^{\prime \prime}, \bar{x}\right)$ corresponds to a vector in $\Delta$ (c.f. Subsection 3.2), we have $d_{B^{\prime \prime}}(\bar{x})=d(\bar{x})=d(x)$ by Lemma 3.5, Theorems 2.5, 2.6 and we are done. 


\section{Orbits of groups generated by symplectic transvections of a linearly independent subset}

In this section, we will prove Theorem 2.15 after some preliminary statements. Throughout the section, $B$ denotes a linearly independent subset which is not a basis in a finite dimensional $\mathbb{F}_{2}$-space $V$ equipped with the alternating form $\Omega$. We always assume that $\operatorname{Gr}(B)$ is connected. We denote by $U$ the linear span of $B$. We note that each $\gamma \in \Gamma_{B}$ preserves cosets in $V / U$, so we only need to describe $\Gamma_{B}$-orbits in each coset $v+U$. If $v+U=U$, then our previous results apply, so we will always consider the action of $\Gamma_{B}$ on a coset $v+U \neq U$. We note that the set $B \cup\{v\}$ is linearly independent and there is the associated graph $\operatorname{Gr}(B \cup\{v\})$ as defined in Section 2. We denote by $V^{\Gamma_{B}}$ the set of vectors in $V$ which are fixed by $\Gamma_{B}$. As before, $U_{0}$ denotes the kernel of the form $\left.\Omega\right|_{U}$ and $\Delta$ is the $\Gamma_{B}$-orbit that contains $B$ ( [1, Proposition 2.1]). The spaces $U_{00}$ and $U_{000}$ are defined as in Section 2.

If $(v+U) \cap V^{\Gamma_{B}}$ is non-empty, then our previous results allows one to describe all $\Gamma_{B}$ orbits in $v+U$. More precisely, we have the following statement.

Proposition 4.1 Suppose that $(v+U) \cap V^{\Gamma_{B}}$ is non-empty and contains a vector $v+u$. Then $\Gamma_{B}$-orbits in $v+U$ are parallel translates of $\Gamma_{B}$-orbits in $U$ by $v+u$.

Our next result gives a sufficient condition for $(v+U) \cap V^{\Gamma_{B}}$ to be empty.

Proposition 4.2 If $\Omega\left(v, U_{000}\right) \neq\{0\}$, then $(v+U) \cap V^{\Gamma_{B}}=\emptyset$.

Proof: We may assume, by Theorems $2.3,2.4$, that $B$ is equivalent to one of the four trees indexed as in figures $2-5$.

Let us introduce the numbers $r$ and $t$ as follows: if $B$ is equivalent to the tree in figure 2 with $m=2$, then $r=m, t=k+1$ and we set $c_{t}=a_{1}$; if $B$ is equivalent to the tree in figure 2 with $m>2$, then $r=m, t=k$; if $B$ is equivalent to the tree in figure 3 or figure 4; then $r=2 n-1, t=p+1$; if $B$ is equivalent to the tree in figure 5 , then $r=2 n, t=p$.

If $\Omega\left(v, U_{000}\right) \neq\{0\}$, then the set $I=\left\{c_{i}: \Omega\left(v, c_{i}\right)=1\right\}$ is a non-empty, proper subset of $\left\{c_{1}, \ldots, c_{t}\right\}$ by Proposition 3.1. We assume, without loss of generality, that $I=\left\{c_{1}, \ldots, c_{s}\right\}, s<t$. Let $x \in U$. If $x$ contains $a_{r}$, then $\tau_{c_{s+1}}(v+x) \neq v+x$. If $x$ does not contain $a_{m}$, then $\tau_{c_{1}}(v+x) \neq v+x$. Thus $v+x \notin V^{\Gamma_{B}}$ for any $x \in U$, i.e. $(v+U) \cap V^{\Gamma_{B}}=\emptyset$

\subsection{Proof of Theorem 2.15.(i)}

We first note that there exist at least two $\Gamma_{B}$-orbits in $v+U$ because, for any $u \in \Delta$ such that $\Omega(u, v)=0$, we have $Q_{B \cup\{v\}}(v+u)=0$, so $v$ and $v+u$ lie in different orbits; here the existence of $u$ follows from our assumption that $\operatorname{dim}(U) \geq 2$.

Since $B$ does not contain any subgraph which is equivalent to $E_{6}$, it is equivalent to a tree of type $D_{m, k}$ with $m \geq 2, k \geq 1$ (Theorem 2.7). Since basic moves preserve the associated quadratic forms (c.f. Section 2), we may take $B$ as in figure 2 with the same indexing. A typical graph of $B \cup v$ is given in figure 19. As a first step, we will disconnect $v$ from $a_{i}$ 's using basic moves. 


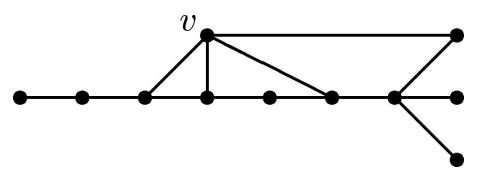

Figure 19. The vertex $v$ is connected to the graph $D_{m, k}, m \geq 2, k \geq 1$.

Lemma 4.3 There exists $\alpha \in \Gamma_{B}$ such that $\Omega\left(\alpha(v), a_{j}\right)=0$ for $j=1, \ldots, m$.

If $m=2$, we set $c_{k+1}=a_{1}$ for convenience. Since $\Omega\left(v, U_{000}\right) \neq\{0\}$, we may assume that $c_{1} \in I=\left\{c_{i}: \Omega\left(v, c_{i}\right)=1\right\}$ by Proposition 3.1.

For any $w \in v+U$, we define $A(w)=\left\{a_{i}: \Omega\left(a_{i}, w\right)=1\right\}$. If $A(w) \neq \emptyset$, we let $i(w)=\max \left\{i: a_{i} \in A(w)\right\}$. Let us write $\alpha_{i}=\tau_{a_{i+1}} \cdots \tau_{a_{m}} \tau_{c_{1}}$ for $i<m$. If $i=i(w)<m$, then $A\left(\alpha_{i}(w)\right)=\left(A(w)-\left\{a_{i}\right\}\right) \cup\left\{a_{i+1}\right\}$, so we have $A\left(\alpha_{m-1} \cdots \alpha_{i+1} \alpha_{i}(w)\right)=(A(w)-$ $\left.\left\{a_{i}\right\}\right) \cup\left\{a_{m}\right\}$. We also note that if $i(w)=m$ and $\Omega\left(w, c_{1}\right)=1$ then $A\left(\tau_{c_{1}}(w)\right)=A(w)-\left\{a_{m}\right\}$. Thus, by induction on $i(v)$ if necessary, we obtain $\alpha \in \Gamma_{B}$ such that $A(\gamma(v))$ is a proper subset of $A(v)$. By induction on the cardinality of $A(v)$, we obtain $\gamma \in \Gamma_{B}$ such that $A(\gamma(v))=\emptyset$. This completes the proof of the lemma.

For the remaining part of the proof of Theorem 2.15.(i) we assume, without loss of generality, that $I=\{b \in B: \Omega(b, v)=1\}=\left\{c_{1}, \ldots, c_{s}\right\}$, where $s<k$ if $m>2$ and $s \leq k+1$ if $m=2$ (here $\left.c_{k+1}=a_{1}\right)$.

Lemma 4.4 Let $f$ be the linear map on the span of $B \cup\{v\}$ defined as follows: $f\left(c_{i}\right)=c_{1}$ for $c_{i}$ in $I$, and $f(b)=b$ for $b \in B \cup\{v\}$ such that $b \notin I$. Then, for any $z \in U$, the vectors $v+z$ and $f(v+z)$ lie in the same $\Gamma_{B}$-orbit.

If $c_{i}$ is not contained in $v+z$ for any $i \in I$, then $f(v+z)=v+z$ and we are done. Let us assume that $v+z$ contains $c_{1}, \ldots, c_{l}$ from $I$. If $v+z$ does not contain $a_{m}$ and $l$ is odd (resp. even), then $f(v+z)=\tau_{c_{l}} \ldots \tau_{c_{2}}(v+z)$ (resp. $f(v+z)=\tau_{c_{l}} \ldots \tau_{c_{1}}(v+z)$ ). Let us now assume that $v+z$ contains $a_{m}$. If $\Omega\left(a_{m}, z\right)=1$ and $l$ is odd (resp. even), then $f(v+z)=\tau_{a_{m}} \tau_{c_{2}} \ldots \tau_{c_{l}} \tau_{a_{m}}(v+z)$ (resp. $\left.f(v+z)=\tau_{a_{m}} \tau_{c_{1}} \ldots \tau_{c_{l}} \tau_{a_{m}}(v+z)\right)$. If $\Omega\left(a_{m}, z\right)=0$ and $l$ is odd (resp. even), then $f(v+z)=\tau_{c_{s+1}} \tau_{a_{m}} \tau_{c_{2}} \ldots \tau_{c_{l}} \tau_{a_{m}} \tau_{c_{s+1}}(v+z)$ (resp. $f(v+z)=$ $\left.\tau_{c_{s+1}} \tau_{a_{m}} \tau_{c_{1}} \ldots \tau_{c_{l}} \tau_{a_{m}} \tau_{c_{s+1}}(v+z)\right)$. This completes the proof of the lemma.

Thus Theorem 2.15.(i) is equivalent to the following statement:

$f(v+x)$ and $f(v+y)$ lie in the same $\Gamma_{B}$-orbit if and only if $Q_{B \cup\{v\}}(f(v+x))=$ $Q_{B \cup\{v\}}(f(v+y))$.

We complete the proof by the following lemma. We recall that $Q_{B}(\alpha(w))=Q_{B}(w)$ for any $w \in V$ and $\alpha \in \Gamma_{B}$.

Lemma 4.5 For any $u \in U$, the vector $f(v+u)$ is in the orbit of either $v$ or $v+a_{m}$.

We first note that $v$ and $v+a_{m}$ lie in different $\Gamma_{B}$-orbits because $Q_{B \cup\{v\}}(v)=1$ and $Q_{B \cup\{v\}}\left(v+a_{m}\right)=0$. To prove the lemma, we will first show that there is $\gamma \in \Gamma_{B}$ such that $\gamma(f(v+u))$ is contained in the chain $A$ formed by $a_{1}, \ldots, a_{m}, c_{1}, v$. Recall that 
$f(v+u)$ does not contain any of $\left\{c_{2}, \ldots, c_{s}\right\}$. Let us first assume that $f(v+u)$ has a component $u_{m}$ that contains $a_{m}$. If $u_{m}$ contains vertices $c_{j_{1}}, \ldots, c_{j_{l}} \subset\left\{c_{s+1}, \ldots, c_{k}\right\}$, then for $\gamma=\tau_{c_{j_{1}}} \ldots \tau_{c_{j,}}, \gamma(f(v+u))$ is contained in $A$. Now let us assume that $u$ does not contain $a_{m}$. If $\Omega\left(a_{m}, f(v+u)\right)=1$, then $\tau_{a_{m}}(f(v+u))=f(v+u)+a_{m}$ contains $a_{m}$ and the previous arguments apply. If $\Omega\left(a_{m}, f(v+u)\right)=0$, then $\tau_{a_{m}} \tau_{c_{1}}(f(v+u))=f(v+u)+c_{1}+a_{m}$ contains $a_{m}$ and we apply the previous arguments.

Thus, we may assume that $f(v+u)=v+f(u)$ is contained in the chain formed by $a_{1}, \ldots, a_{m}, c_{1}, v$. We may also assume that $\Omega(v, f(u))=0$, (otherwise we can write $f(v+u)=c_{1}+a_{m}+a_{m-1}+\cdots+a_{m-i}+x$ where $x \in \operatorname{span}\left(a_{1}, \ldots, a_{i}\right)$ and consider $\tau_{c_{1}} \tau_{a_{m}} \tau_{a_{m-1}} \ldots \tau_{a_{m-i}}(f(v+u))$. Then, by Theorem 2.5, there exists $\beta \in \Gamma_{\left\{a_{1}, \ldots, a_{m}\right\}}$ such that $\beta(f(v+u))=v+a_{m}+a_{m-2}+\cdots+a_{m-2 r}$ for some $r \geq 0$. If $r=0$, then we are done. We suppose $r \geq 1$. Then, for $\alpha=\tau_{c_{1}} \tau_{a_{m}} \tau_{a_{m-1}} \tau_{c_{s+1}} \tau_{a_{m}} \tau_{c_{1}} \tau_{a_{m-2}} \tau_{a_{m-1}} \tau_{a_{m}} \tau_{c_{s+1}}$, we have $\alpha\left(v+a_{m}+a_{m-2}+\cdots+a_{m-2 r}\right)=v+a_{m-4}+\cdots+a_{m-2 r}$, which has two less components than $\beta(f(v+u))=v+a_{m}+a_{m-2}+\cdots+a_{m-2 r}$. Continuing this process, we will have $f(v+x)$ in the orbit of either $v$ or $v+a_{m}$, which proves the lemma. This also completes the proof of Theorem 2.15.(i).

\subsection{Proof of Theorem 2.15.(ii)}

If $(v+U) \cap V^{\Gamma_{B}} \neq \emptyset$, then the statement follows from Proposition 4.2. Let us now assume that $(v+U) \cap V^{\Gamma_{B}} \neq \emptyset$. By Theorem 2.7, we may take $B$ as in figure 2 with the same indexing. If $\Omega\left(v, U_{000}\right)=\{0\}$, then $v$ is connected to none of the $c_{i}$ 's or connected to all of them as in figures 20 and 21. If $v$ is connected to all of $c_{i}$ 's, then $v+a_{m}$ is connected to only $a_{i}$ 's, so we may assume that $v$ is connected only to $a_{i}$ 's. Suppose $v$ is connected to $a_{i}$, i.e. $\Omega\left(v, a_{i}\right)=1$, but $\Omega\left(v, a_{j}\right)=0$ for $j=i+1, \ldots, m$. Then $v+a_{i-1}$ will not be connected to $a_{i}, \ldots, a_{m}$. Continuing this way, we will have a $w=v+u$ connected to only $a_{1}$. Thus, $B \cup\{w\}$ will be of type $D_{m+1, k}$.

Let us now prove that,

for $x, y \in U$, the vectors $w+x$ and $w+y$ lie in the same $\Gamma_{B}$-orbit

if and only if they lie in the same $\Gamma_{B \cup\{w\}}$-orbit.

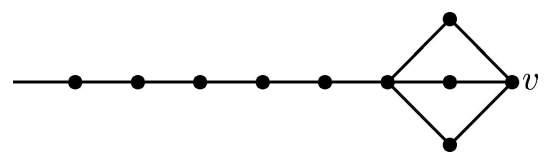

Figure 20. The vertex $v$ is connected to all $c_{i}$ 's.

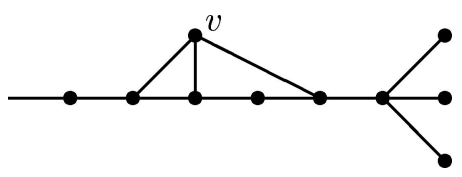

Figure 21. The vertex $v$ is connected to none of the $c_{i}$ 's. 
The "only if" part follows from the fact that $\Gamma_{B}$ is a subgroup of $\Gamma_{B \cup\{w\}}$. To prove the "if" part, we assume, by Theorem 2.5, that $f(w+x)$ and $f(w+y)$ have the same number of connected components. Then it is easy to see the following: there is $\alpha, \beta \in \Gamma_{B}$ such that $\alpha(w+x)=w+x^{\prime}, \beta(w+y)=w+y^{\prime}$ with $\Omega\left(w, x^{\prime}\right)=0$ and $\Omega\left(w, y^{\prime}\right)=0$. We will show that $w+x^{\prime}$ and $w+y^{\prime}$ lie in the same $\Gamma_{B}$-orbit. We note that $x^{\prime}, y^{\prime} \in$ $S=\operatorname{span}\left(a_{2}, \ldots, a_{m}, c_{1}, \ldots, c_{k}\right)$ and $f\left(x^{\prime}\right)$ and $f\left(y^{\prime}\right)$ have the same number of connected components). Thus there exists $\gamma \in \Gamma_{S}$ such that $\gamma\left(x^{\prime}\right)=y^{\prime}$. Since $\Omega(w, s)=0$ for any $s \in S$, we have $\gamma\left(w+x^{\prime}\right)=w+y^{\prime}$ and we are done.

Example 4.6 For this example, let $B=\left\{b_{1}, b_{2}, b_{3}, b_{4}, b_{5}, b_{6}\right\}$ and let $V$ denote the vector space over $\mathbb{F}_{2}$ with basis $B \cup\left\{v_{1}, v_{2}, v_{3}, v_{4}\right\}$. We denote by $\Omega$ the skew-symmetric form given in figure 22. As above, $U$ denotes the linear span of $B$ in $V$. We determine $\Gamma_{B}$-orbits in $V$ as follows:

It is easy to see that $c=b_{2}+b_{4}+b_{6} \in U_{000}$. Let $V_{1,4}$ denote the linear span of the set $\left\{v_{1}, v_{4}\right\}$. Since $\Omega\left(v_{2}, c\right)=\Omega\left(v_{3}, c\right)=1$ and $\Omega\left(v_{1}, c\right)=\Omega\left(v_{4}, c\right)=0$, by Theorem 2.15, we have the following:

If $v \in v_{2}+V_{1,4}$ or $v \in v_{3}+V_{1,4}$, then the $\Gamma_{B}$-orbits in the coset $v+U$ are intersections of $v+U$ with the sets $Q_{B \cup\{v\}}^{-1}(0)$ and $Q_{B \cup\{v\}}^{-1}(1)$ (so there are 16 of such orbits).

The remaining $\Gamma_{B}$-orbits are contained in cosets $v+U$ where $v \in S=\operatorname{span}\left(\left\{v_{2}+\right.\right.$ $\left.\left.v_{3}, v_{1}, v_{4}\right\}\right)$.

To proceed, we first notice that $v_{2}+v_{3}+b_{5}$ and $v_{1}+v_{4}+b_{6}+b_{5}$ are fixed by $\Gamma_{B}$. Now we note the following fact: for $v, w \in V$, if $v+w$ is fixed by $\Gamma_{B}$, then $\Gamma_{B}$-orbits in $v+U$ are parallel translates of $\Gamma_{B}$-orbits in $w+U$ by $v+w$ (because $\alpha(v+u)=$ $\alpha(v+w+w+u)=v+w+\alpha(w+u)$ for all $\left.\alpha \in \Gamma_{B}, u \in U\right)$. We also note that the one-element $\Gamma_{B}$-orbits in $U$ are the vectors $\left\{0, b_{2}+b_{6}+b_{4}, b_{1}+b_{5}+b_{3}+b_{4}, b_{1}+\right.$ $\left.b_{5}+b_{3}+b_{2}+b_{6}\right\}$. The non-trivial $\Gamma_{B}$-orbits have representatives $b_{5}$ and $b_{5}+b_{6}$. Also all $\Gamma_{B}$-orbits in $v_{1}+U$ are non-trivial and they have representatives $v_{1}, v_{1}+b_{5}, v_{1}+b_{5}+b_{6}$. Thus, the total number of $\Gamma_{B}$-orbits is $16+4 \cdot 6+4 \cdot 3=16+24+12=52$.

According to [24], there is a bijection between $\Gamma_{B}$-orbits and connected components of the reduced double Bruhat cell $L^{w_{0}, e}(\mathbb{R})$ for $W=S_{5}$ and

$w_{0}=s_{1} s_{3} s_{2} s_{4} s_{1} s_{3} s_{2} s_{4} s_{1} s_{3}$

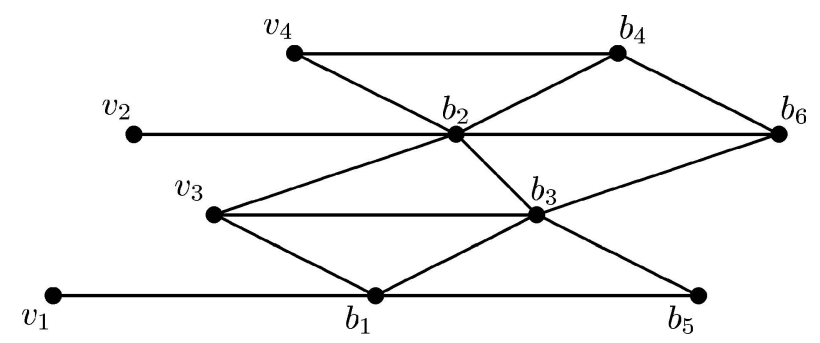

Figure 22. The graph for $\Omega$ in Example 4.6. (Example 4). 
where $s_{i}=(i, i+1)$ are adjacent transpositions. We note that $w_{0}$ is the longest element of $W$ and $\mathbf{i}=(1,3,2,4,1,3,2,4,1,3)$ is its reduced word and the set of bounded indices $B$ (i) (see [24]) is $B$. We remark that the total number of $\Gamma_{B}$-orbits agrees with the result given in [22].

\section{Orbits of groups generated by non-symplectic tranvections}

In this section, we prove Theorem 2.16 and give an example. One could easily note that Theorem 2.16 follows from the following lemma which extends [8, Theorem 3] to an arbitrary bilinear form.

Lemma 5.1 Let $V$ be an $\mathbb{F}_{2}$ space equipped with a non-skew-symmetric bilinear form $\Omega(u, v)$. Suppose that $B$ is a linearly independent subset of $V$ such that $\Omega(b, b)=0$ for all $b \in B$ and $G r(B)$ is connected. Let $x$ be a vector in $V$ and let $B_{L}$ be an arbitrary connected subgraph such that the group $\Gamma_{B_{L}}$ does not fix $x$. Suppose that $b \in B-B_{L}$ and let $P=$ $\left[b_{0} \in T(x), \ldots, b_{k}=b\right]$ be a shortest path that connects $b$ to $T(x)=b \in B_{L}: \tau_{b}(x) \neq x$. If $\left.\Omega\right|_{P_{L}(b, x)}$ is not alternating and $\Omega\left(b_{i}, b_{i+1}\right)=1$ for $i=0, \ldots, k-1$, then the vector $x+b$ lies in the $\Gamma_{B}$ orbit that contains $x$.

We prove the lemma by modifying the proof of [8, Theorem 3]. Let us first note that $\Omega\left(b_{i}, b_{j}\right)=0$ for $0 \leq i<i+1<j \leq k$ because $P$ is a shortest path. Let $l=\min \{i \geq 1$ : $\Omega\left(b_{i-1}, b_{i}\right)=1$ but $\left.\Omega\left(b_{i}, b_{i-1}\right)=0\right\}$.

We will prove the lemma by induction on $k \geq 0$, the length of $P$. The case $k=0$ is clear: $\tau_{b}(x)=x+b$. Suppose that the statement of the lemma holds for $P$ of length less than $k$. Let us first assume that $\tau_{b_{j}}(x)=x$ for $j=l, \ldots, k$. Then $\tau_{b_{k}} \ldots \tau_{b_{0}}(x)=$ $x+b_{0}+\cdots+b_{l-1}+b_{l}+\cdots+b_{k}$. Since $\tau_{b_{l-1}}\left(x+b_{0}+\cdots+b_{k}\right)=\left(x+b_{0}+\cdots+b_{k}\right)+b_{l-1}$, we have

$$
\tau_{b_{k}} \ldots \tau_{b_{l}} \tau_{b_{0}} \ldots \tau_{b_{l-1}} \tau_{b_{k}} \ldots \tau_{b_{0}}(x)=x+b
$$

Let us now assume that there exists $l-1<j<k+1$ such that $\tau_{b_{j}}(x) \neq x$ and let $m$ be the one closest to $b_{k}=b$. We note that $z=\tau_{b_{k}} \ldots \tau_{b_{m}}(x)=x+b_{m}+\cdots+b_{k}$ is in the same orbit as $x$. Then, the length of the shortest path connecting $T(z)$ to $b_{k-1}$ is less than $k$, hence by the induction hypothesis, $z+b_{k-1}=x+b_{m}+\cdots+b_{k-2}$ lies in the orbit of $x$. Applying the same procedure to $b_{k-2}, \ldots, b_{m}$, we will have $x+b_{k}$ in the orbit of $x$.

Example 5.2 Let $V$ be the vector space over $\mathbb{F}_{2}$ with basis $B^{\prime}=\left\{b_{1}, \ldots, b_{6}, v_{1}, v_{2}, v_{3}\right\}$. Let us introduce the following sets: $B=\left\{b_{1}, b_{2}, b_{3}, b_{4}, b_{5}, b_{6}\right\}, B_{R}=\left\{b_{1}, b_{2}, b_{4}, b_{5}\right\}$, $B_{L}=\left\{b_{3}, b_{6}\right\}, C_{R}=\left\{v_{1}, v_{2}\right\}, C_{L}=\left\{v_{3}\right\}, R=B_{R} \cup C_{R} L=B_{L} \cup C_{L}$. We denote by $\Omega$ the bilinear form on $V$ defined by $\operatorname{Gr}\left(B^{\prime}\right)$ given in figure 23 such that $\left.\Omega\right|_{\mathbb{F}_{2}^{R}}$ and $\left.\Omega\right|_{\mathbb{F}_{2}^{L}}$ are alternating and $\Omega\left(a_{r}, a_{l}\right)=0$ for any $a_{r} \in R$ and $a_{l} \in L$.

To determine the $\Gamma_{B}$-orbits in $V$, we first note that the vectors $\left\{b_{1}+b_{2}+b_{4}, b_{1}+b_{5}, b_{2}+\right.$ $\left.b_{4}+b_{5}\right\} \subset\left(\mathbb{F}_{2}^{B_{U}}\right)$. Let us write $V_{1,2}=$ linear span of $\left\{v_{1}, v_{2}, v_{3}+b_{6}\right\}$, which is the set of fixed points of $\Gamma_{B_{L}}$. Then for any $v \in V_{1,2}$ such that $v \notin\left\{0, v_{3}+v_{1}+b_{6}\right\}$, we have 


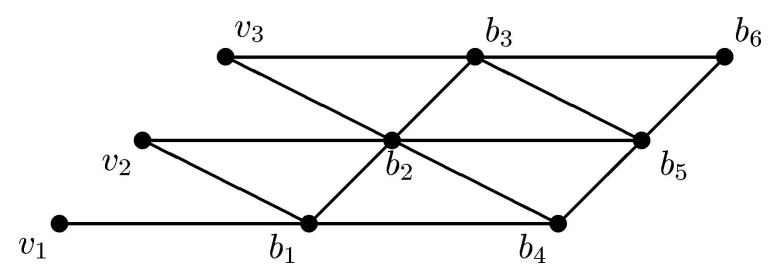

Figure 23. The graph for $\Omega$ in Example 5.2. (Example 5).

$\Omega\left(v,\left(\mathbb{F}_{2}^{B_{R}}\right)_{000}\right) \neq 0$. Thus, by Proposition 2.16 and Theorem 2.15, we have the following: For any $v \in V_{1,2}$ such that $v \notin\left\{0, v_{3}+v_{1}+b_{6}\right\}$, the $\Gamma_{B}$-orbits in the coset $v+\mathbb{F}_{2}^{B_{R}}$ are intersections of $v+\mathbb{F}_{2}^{B_{R}}$ with the sets $Q_{B \cup\{v\}}^{-1}(0)$ and $Q_{B \cup\{v\}}^{-1}(1)$ (so there are 12 such orbits). We also note that $v_{3}+v_{1}+b_{6}+b_{4}$ is fixed by $\Gamma_{B_{R}}$, thus the $\Gamma_{B_{R}}$ (hence $\Gamma_{B}$ )orbits in $v_{3}+v_{1}+b_{6}+\mathbb{F}_{2}^{B_{R}}$ are parallel translates of $\Gamma_{B_{R}}$-orbits in $\mathbb{F}_{2}^{B_{R}}$ (Proposition 4.1). The one-element $\Gamma_{B_{R}}$-orbits in $\mathbb{F}_{2}^{B_{R}}$ are $0, b_{2}+b_{4}+b_{5}, b_{2}+b_{4}+b_{1}, b_{1}+b_{5}$ and the remaining one is represented by $b_{1}$, so there is a total of $10 \Gamma_{B}$-orbits in $v+\mathbb{F}_{2}^{B_{R}}$ for any $v \in\left\{0, v_{3}+v_{1}+b_{6}\right\}$. According to Theorem 2.16, the remaining orbits are represented by the vectors $v_{3}, v_{3}+v_{1}, v_{3}+v_{2}, v_{3}+v_{1}+v_{2}, b_{3}, b_{3}+v_{1}, b_{3}+v_{2}, b_{3}+v_{1}+v_{2}$.

Thus, the total number of $\Gamma_{B}$-orbits is $12+10+8=30$.

According to [28], $\Gamma_{B}$-orbits are in bijection with the connected components of the reduced double Bruhat cell $L^{w_{0}, e}(\mathbb{R})$ for $W$ of type $B_{3}$, where $w_{0}$ is the longest element in its Weyl group. We remark that the total number of $\Gamma_{B}$-orbits agrees with the result obtained in [8].

\section{Acknowledgments}

I am grateful to my graduate advisor Andrei Zelevinsky for his support and many suggestions. I also thank Misha Kogan and Josh Scott for helpful discussions.

\section{References}

1. R. Brown and S. Humphries, "Orbits under symplectic transvections I," Proc. London Math. Soc. 52(3) (1986), 517-531.

2. R. Brown and S. Humphries, "Orbits under symplectic transvections II: The case $K=F_{2}$," Proc. London Math. Soc. 52(3) (1986), 532-556.

3. H. Cárdenas, E. Lluis, Raggi-Cárdenas, A. Gerardo, and R. San Agustín, "Partial linear spaces with dual affine planes," Comm. Algebra 30(2) (2002), 603-617.

4. R.W. Carter, Simple Groups of Lie Type, Wiley, London, 1972.

5. J. Dieudonne, La geometrie des groupes classiques, Springer, Berlin, Gottingen, Heidelberg, 1955.

6. W. Ebeling, "The monodromy groups of isolated singularities of complete intersections," Lecture Notes in Mathematics, 1293, Springer-Verlag, Berlin, 1987.

7. S. Fomin and A. Zelevinsky, "Double Bruhat cells and total positivity," J. Amer. Math. Soc. 12 (1999), 335-380.

8. M. Gekhtman, M. Shapiro, and A. Vainshtein, "The number of connected components in the double Bruhat cells for non simply-laced groups," Proc. Amer. Math. Soc. 131(3), (2003), 731-739. 
9. J.I. Hall, "Symplectic geometry and mapping class groups. Geometrical combinatorics (Milton Keynes, 1984)," Res. Notes in Math., 114, Pitman, Boston, MA, 1984, pp. 21-33.

10. J.I. Hall, "Graphs, geometry, 3-transpositions, and symplectic $F_{2}$-transvection groups," Proc. London Math. Soc. (3) 58(1), (1989), 89-111.

11. J.I. Hall, "Some 3-transposition groups with normal 2-subgroups.” Proc. London Math. Soc. (3) 58 (1989)(1), $112-136$.

12. R.C. Hamelink, "Lie algebras of characteristic 2." Trans. Amer. Math. Soc. 144 (1969), 217-233.

13. T. Hoffmann, J. Kellendonk, N. Kutz and N. Reshetikhin, "Factorization dynamics and Coxeter-Toda lattices," Comm. Math. Phys. 212(2) (2000), 297-321.

14. S. Humphries, "Graphs and Nielsen transformations of symmetric, orthogonal and symplectic groups," Quart. J. Math. Oxford, 36(2) (1985), 297-313.

15. W.A.M. Janssen, "Skew-symmetric vanishing lattices and their monodromy groups," Math. Ann. 266 (1983), $115-133$.

16. M. Kogan and A. Zelevinsky, "On symplectic leaves and integrable systems in standard complex semisimple Poisson-Lie groups," Intern. Math. Res. Notices 32 (2002), 1685-1702.

17. G. Lusztig, "Total positivity in reductive groups," in "Lie theory and geometry: in honor of B. Kostant", Progr. in Math.123, Birkhauser (1994), 531-568.

18. J. McLaughlin, "Some groups generated by transvections.” Arch. Math. (Basel) 18(1967), 364-368.

19. J. McLaughlin, "Some subgroups of $\mathrm{SL}_{n}\left(F_{2}\right)$." Illinois J. Math. 13(1969), 108-115.

20. J.J. Rotman and P.M. Weichsel, "Simple Lie algebras and graphs," J. Algebra 169(3) (1994), 775-790.

21. J.J. Seidel, On two-graphs and Shult's characterization of symplectic and orthogonal geometries over GF(2). T.H.-Report, No. 73-WSK-02. Department of Mathematics, Technological University Eindhoven, Eindhoven, 1973. $i+25$.

22. B. Shapiro, M. Shapiro, and A. Vainshtein, "Connected components in the intersection of two open opposite Schubert cells in $S L_{n}(\mathbb{R}) /$ B," Internat. Math. Res. Notices, 10(1997), 469-493.

23. B. Shapiro, M. Shapiro, and A. Vainshtein, "Skew-symmetric vanishing lattices and intersections of Schubert cells,” Internat. Math. Res. Notices, 11(1998), 563-588.

24. B. Shapiro, M. Shapiro, A. Vainshtein, and A. Zelevinsky, "Simply-laced Coxeter groups and groups generated by symplectic transvections," Michigan Mathematical Journal, 48(2000), 531-552.

25. E.E. Shult, "Characterizations of certain classes of graphs," J. Combinatorial Theory Ser. B 13(1972), 142167.

26. E.E. Shult, Groups, polar spaces and related structures. Combinatorics, Part 3: Combinatorial group theory (Proc. Advanced Study Inst., Breukelen, 1974), Math. Centre Tracts, Math. Centrum, Amsterdam, 57(1974), $130-161$.

27. B. Wajnryb, "On the monodromy group of plane curve singularities," Math. Ann. 246(1980), 141-154.

28. A. Zelevinsky, "Connected components of real double Bruhat cells," Intern. Math. Res. Notices 21(2000), 1131-1153. 